Check for updates

Cite this: RSC Adv., 2018, 8, 732

Received 8th November 2017

Accepted 18th December 2017

DOI: $10.1039 / c 7 r a 12246 b$

rsc.li/rsc-advances

\section{Three successive and regiocontroled palladium cross-coupling reactions to easily synthesize novel series of 2,4,6-tris(het)aryl pyrido[1',2':1,5]pyrazolo $[3,4-d]$ pyrimidines $\dagger$}

\author{
R. Belaroussi, ${ }^{\text {ab }}$ A. Ejjoummany, ${ }^{\text {ab }}$ A. El Hakmaoui, ${ }^{b}$ M. Akssira, ${ }^{\text {b }}$ G. Guillaumet (iD *a \\ and S. Routier (iD *a
}

\begin{abstract}
The first access to tris(het)arylated pyrido $\left[1^{\prime}, 2^{\prime}: 1,5\right]$ pyrazolo[3,4-d]pyrimidine derivatives is reported. The series were generated from 4-chloroaminopyridinium, which afforded the key intermediate bearing three leaving groups, i.e. a C-2 methylsulfanyl, a lactame carbonyl group in C-4 and a chlorine atom in C-6. The regioselective reactions led to the tris(het)aryl derivatives with satisfying to high yields. The three successive cross-coupling reactions occurred first in C- 6 by the displacement of chlorine, next in C-4 position by a sequential Pd-catalyzed phosphonium coupling and finally in C-2 under a Pd/Cucatalyzed desulfitative cross-coupling reaction. The optimization and scope of each reaction are discussed and the original compounds characterized.
\end{abstract}

\section{Introduction}

Nitrogen-containing and fused heterocyclic compounds have attracted considerable attention in directed and targeted organic synthesis and many products have been used in materials and medicinal chemistry programs. With a view to increasing molecular diversity, our aim was to design rare tricyclic pyrido[ $\left[1^{\prime}, 2^{\prime}: 1,5\right]$ pyrazolo[3,4- $\left.d\right]$ pyrimidines (PPPy), which incorporate a tricyclic system and four nitrogen atoms, two of which are vicinal. This fused tricyclic heterocycle contains a pyrazolopyridine core which has been used in diverse biologically and pharmaceutical active molecules including diuretic $^{1}$ and antiherpetic drugs, ${ }^{2}$ and as p38 kinase inhibitors ${ }^{3}$ and melatonine receptor ligands. ${ }^{4}$ It also contains a pyrimidine natural moiety, which is known to possess a potential role in several biological processes and to have emerged as a significant motif in drug-discovery programs. ${ }^{5}$

In order to build such a multi-heterocyclic compound, our group recently focused on developing new functionalization protocols $^{6}$ to explore the chemical space, an indispensable key to design future useful molecules.

${ }^{a}$ Univ Orleans, CNRS, Institut de Chimie Organique et Analytique, UMR 7311, BP 6759, 45067 Orléans Cedex 2, France. E-mail: sylvain.routier@univ-orleans.fr; gerald. guillaumet@univ-orleans.fr; Web: http://www.icoa.fr/fr

${ }^{b}$ Laboratoire de Chimie Physique et Chimie Bioorganique, FST Mohammedia, Université Hassan II de Casablanca, B. P. 146, 28800 Mohammedia, Morocco. E-mail: akssira@yahoo.fr; Web: http://www.fstm.ac.ma

$\dagger$ Electronic supplementary information (ESI) available. See DOI: $10.1039 / \mathrm{c} 7 \mathrm{ra} 12246 \mathrm{~b}$

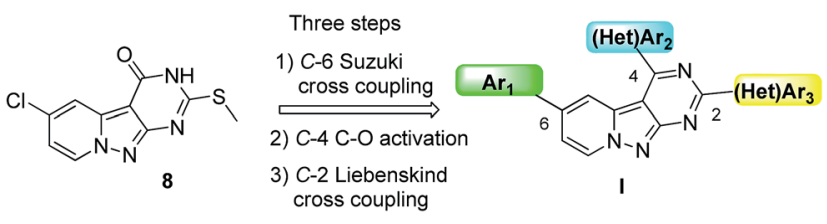

Fig. 1 Access to 2,4,6-tris(het)arylated pyrido $\left[1^{\prime}, 2^{\prime}: 1,5\right]$ pyrazolo $[3,4-d]$ pyrimidines I from the unique platform 8 .

In order to build C-2 and C-4 disubstituted PPPy derivatives, we developed a straightforward strategy which included successive $\mathrm{C}-4$ in situ $\mathrm{C}-\mathrm{O}$ activation and a $\mathrm{C}-2$ desulfurization through Liebeskind-Srogl cross-coupling reactions. ${ }^{7}$ To provide a versatile platform we then sought to design a new flexible route to access 2,4,6-trisubstituted PPPy's I from a unique intermediate i.e. the 6 -chloro-2-(methylthio)pyrido $\left[1^{\prime}, 2^{\prime}: 1,5\right]$ pyrazolo[3,4- $d]$ pyrimidin-4(3H)-one 8 .

We report herein the preparation of $\mathbf{8}$ and its regioselective functionalization by different types of palladium catalyzed reactions. After optimization of the reaction conditions, this strategy was achieved using three successive (het)arylations in the following order C-6, C-4 and then C-2 positions (Fig. 1). The scope and limits are discussed for each step.

\section{Results and discussion}

Compound 8 was obtained via a 7-step sequence partially based on our previous work. ${ }^{7,8}$ Dimethyl 5-chloropyrazolo[1,5-a] pyridine-2,3-dicarboxylate 2 was obtained after a [3+2] cycloaddition between the 1-amino-4-chloropyridinium iodide 1 
(ref. 9 and 10) with dimethyl acetylenedicarboxylate (DMAD). A regioselective saponification furnished the hemiester 3 , which was subjected to an isocyanate generation under Curtius rearrangement. The unstable isocyanate function was quenched with tert-butanol to afford the Boc protected (het)aryl amine 4. After removal of the carbamate and benzoylisothiocyanate condensation, the thiourea 6 was subjected to annelation and finally a regioselective sulfur methylation led to $\mathbf{8}$ in satisfying yields (Scheme 1). This methodology proved to be highly efficient and amenable to multigram scale to access the unique and challenging tricyclic structure in sufficient amounts to pursue the optimization of the (het)arylation process.

\section{Suzuki-Miyaura arylation in C-6}

The functionalization of the $\mathrm{C}-4$ position of compound $\mathbf{8}$ was first attempted via a $\mathrm{C}-\mathrm{O}$ direct activation involving $\mathrm{PyBrOP}^{11}$ to generate the required in situ generated $O$-phosphonium leaving group (Scheme 2). However, this route proved to be unsuitable since only the disubstituted product 9 was isolated in $45 \%$ yield with no trace of the mono C-4-arylated molecule. This result seems to indicate that the $6-\mathrm{Cl}$ atom is as reactive as the activated $4-\mathrm{O}$ atom under palladium catalyzed conditions.

In an attempt to selectively substitute one of the two positions (C-6 or C-4), we began by the regioselective functionalization of the C-6 position. Using several experimental coupling Suzuki-Miyaura conditions exploited in our previous research, ${ }^{12}$ this first test did not allow the formation of the $\mathrm{C}-\mathrm{C}$ bond, as only the starting material 8 was recovered (Table 1 , entry 1 ). The same behavior was observed using the conditions reported in Scheme 2, as if pyrimidinone exerted a negative impact on C-6 reactivity in basic and aqueous media.

To circumvent this problem, we switched the palladium source and tested the catalytic system XantPhos $/ \mathrm{Pd}(\mathrm{OAc})_{2} \cdot{ }^{13}$ The reaction was carried out in dioxane under microwave irradiation at $150{ }^{\circ} \mathrm{C}$ using potassium carbonate as a base. To our delight these conditions provided the desired product $\mathbf{1 0}$ in $63 \%$ yield (Table 1 , entry 3 ).

Next, the generalization of this approach was examined (Table 2). Results were found to be satisfactory as the majority of the boronic substrates employed provided the coupling products. Electron rich or deactivated aryl as well as naphthyl
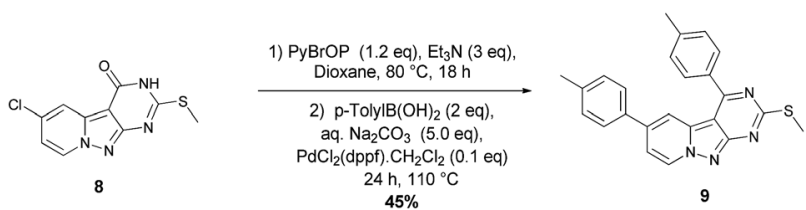

Scheme 2 Phosphonium-mediated Suzuki type cross coupling reaction from 8 .

residues were introduced with good yields ranging from 56 to $73 \%$. In all cases a full conversion of starting material and formation of a unique product were observed. The decrease in yield was mainly due to difficulties encountered during purification steps.

\section{2. (Het)arylations under direct $\mathrm{C}-\mathrm{O}$ activation in $\mathrm{C}-4$}

After having regioselectively introduced the C-6 aryl group, the direct C-4 arylation via PyBrOP amid activation was undertaken, applying the operating conditions previously established for the synthesis of 9. ${ }^{7}$ The reaction sequence involved first the activation of the $\mathrm{C}-\mathrm{O}$ bond using $\mathrm{Et}_{3} \mathrm{~N}$ to favor the formation of the oxanion which was in situ quenched by PyBrOP (1.2 eq.) activating agent. The second step, which required the addition of boronic acid and $\mathrm{PdCl}_{2}(\mathrm{dppf}) \cdot \mathrm{CH}_{2} \mathrm{Cl}_{2}$ as catalyst, was successfully achieved under thermal conditions. Initially, we carried out our investigations on 10, 11 and $\mathbf{1 3}$ (Table 3), which carry an electro-donor aryl group in C-6. The coupling reactions were achieved with representative boronic acids. Aryl and thiophenyl groups were easily introduced (entries 1, 4, 5, 7, 8, 9). The decreases in yield of purified product are mainly due to the purification step. Some difficulties occurred with the deactivated fluorophenyl and pyridinyl groups (entries 2, 6). The reactions appear incomplete and the corresponding starting materials were recovered. All modifications of the experimental conditions were inefficient. In the pyridinyl series, the introduction of a strong electrodonor OMe group restored the reactivity (entry 3 ).

While all reactions were inefficient starting from 15 (entry 11), we were able to introduce the 4-cyanophenyl group on $\mathbf{1 4}$ (entry 10) and derivative 25 was formed with an average yield of $40 \%$ whereas $21 \%$ of starting material still remained

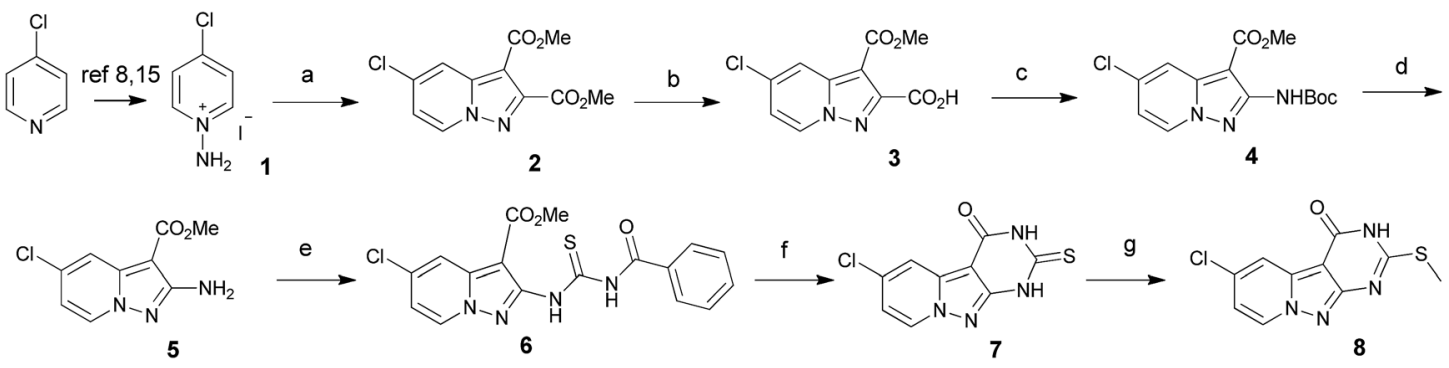

Scheme 1 Synthetic route for 8: (a) DMAD (1.5 eq.), $\mathrm{K}_{2} \mathrm{CO}_{3}$ (1.4 eq.), DMF, r.t., $12 \mathrm{~h}, 86 \%$; (b) $\mathrm{NaOH}$ (2N) $\left(1.0\right.$ eq.), $\mathrm{CH}_{3} \mathrm{OH}, \mathrm{r.t.}, 6$ h, $81 \%$; (c) (i) Et ${ }_{3} \mathrm{~N}$ (1.3 eq.), $\mathrm{ClCO}_{2} \mathrm{Et}\left(1.5\right.$ eq.), THF, $-10{ }^{\circ} \mathrm{C}, 1.5 \mathrm{~h}$, (ii) $\mathrm{NaN}_{3}$ (1.7 eq.), $-10{ }^{\circ} \mathrm{C}, 1.5 \mathrm{~h}$, (iii) $t$-butanol, $6 \mathrm{~h}, 67 \%$; (d) $\mathrm{TFA} / \mathrm{CH}_{2} \mathrm{Cl}_{2}(1 / 1), 4$ h, r.t., quant.; (e) benzoylisothiocyanate (1.2 eq.), $\mathrm{CHCl}_{3}, 12$ h, r.t., 84\%; (f) EtONa (1.1 eq.), EtOH, 8 h, reflux, 97\%; (g) Mel (1.0 eq.), $\mathrm{NaOH}$ (1.0 eq.), EtOH, 6 h, r.t., $80 \%$. 
Table 1 Suzuki-Miyaura conditions for C -6 reaction of 8

\begin{tabular}{|c|c|c|c|}
\hline Entry & Conditions & & $\begin{array}{l}\text { Yield in } \\
10(\%)\end{array}$ \\
\hline 1 & \multirow{3}{*}{\multicolumn{2}{|c|}{$\begin{array}{l}p \text {-TolylB(OH })_{2}\left(1.2 \text { eq.), DME/ } \mathrm{H}_{2} \mathrm{O}(2 / 1), \mathrm{Na}_{2} \mathrm{CO}_{3}\left(2 \text { eq.), } \mathrm{Pd}\left(\mathrm{PPh}_{3}\right)_{4}(5 \% \mathrm{~mol}), 30 \mathrm{~min}, \mathrm{M} . \mathrm{W}, 120{ }^{\circ} \mathrm{C}\right.\right. \\
p \text {-TolylB(OH })_{2} \text { (1.1 eq.), 1,4-dioxane/ } \mathrm{H}_{2} \mathrm{O}(4 / 1), \mathrm{Na}_{2} \mathrm{CO}_{3}(3.0 \text { eq. }), \mathrm{PdCl}_{2}(\mathrm{dppf}) \cdot \mathrm{CH}_{2} \mathrm{Cl}_{2}(10 \% \mathrm{~mol}), 1 \mathrm{~h} / \mathrm{M} . \mathrm{W}, 150{ }^{\circ} \mathrm{C} \\
p \text {-TolylB(OH })_{2}\left(1.1 \text { eq.), } 1,4 \text {-dioxane, } \mathrm{K}_{2} \mathrm{CO}_{3}(2.0 \text { eq. }), \mathrm{Pd}(\mathrm{OAc})_{2} / \mathrm{xantphos}(10 \% / 20 \% \mathrm{~mol}), 1 \mathrm{~h} / \mathrm{M} . \mathrm{W}, 150{ }^{\circ} \mathrm{C}\right.\end{array}$}} & $\mathrm{ND}^{a}$ \\
\hline 2 & & & $\mathrm{ND}^{a}$ \\
\hline 3 & & & $63^{b}$ \\
\hline
\end{tabular}

Table 2 Exemplification of Suzuki-Miyaura cross coupling in C-6 from 8

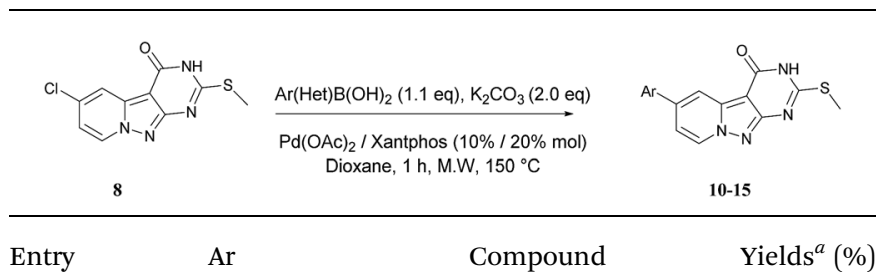

\begin{tabular}{llll} 
Entry & Ar & Compound & Yields $^{a}(\%)$ \\
\hline
\end{tabular}

1<smiles>Cc1ccc(C2CC2)cc1</smiles>

\section{0}<smiles>Cc1ccc([O-])cc1</smiles>

11

63

2<smiles>COc1cccc(C(C)(C)C)c1</smiles><smiles>c1ccc2cc(CC3CCCC3)ccc2c1</smiles>

13

14

15<smiles>CCc1ccc(C(C)(C)C)cc1</smiles>

66

56

${ }^{a}$ Yields are indicated as isolated compounds.

unchanged after $24 \mathrm{~h}$. In order to improve the overall conversion, a longer reaction time ( $72 \mathrm{~h})$, was used but degradation occurred.

Based on the results obtained, we can conclude that the electronic properties of the C- 6 aryl moieties strongly affect the reactivity of the $\mathrm{C}-4$ position. The best conversions were always obtained with the simultaneous presence of electron donor substituents (mesomeric or inductive effect) on the C-6 position as well as on the boronic acid used.

\section{3. (Het)arylations under Liebeskind-Srogl in C-2}

We next applied the third Liebeskind-Srogl ${ }^{14}$ method to the 4,6bis(het)arylated derivatives in an attempt to furnish original
Table 3 Exemplification of amid direct arylation in C-4

\begin{tabular}{|c|c|c|c|c|}
\hline & ${ }^{0}-\mathrm{NH}$ & \multicolumn{2}{|c|}{$\begin{array}{l}\text { 1) PyBroP (1.2 eq), } \mathrm{Et}_{3} \mathrm{~N}(3.0 \mathrm{eq}) \\
\text { Dioxane, } 80^{\circ} \mathrm{C}, 18 \mathrm{~h} \\
\mathrm{R}_{2}-\mathrm{B}\left(\mathrm{OH}_{2}(2.0 \mathrm{eq}), \mathrm{Na}_{2} \mathrm{CO}_{3}\left(\mathrm{H}_{2} \mathrm{O}\right)(5.0 \mathrm{eq})\right. \\
\mathrm{PdCl}_{2}(\mathrm{dppf}) \mathrm{CH}_{2} \mathrm{Cl}_{2}(0.1 \mathrm{eq}) \\
\text { Dioxane, } 110^{\circ} \mathrm{C}, 24 \mathrm{~h}\end{array}$} & $\mathrm{R}_{1}^{\mathrm{R}_{2}}=\mathrm{N}$ \\
\hline Entry & Substrate & Boronic acids & Product & Yield $^{a}(\%)(\mathrm{SM} \%)^{b}$ \\
\hline 1 & & $(\mathrm{HO})_{2} \mathrm{~B}-$ & 16 & 55 \\
\hline 2 & 10 & $(\mathrm{HO})_{2} \mathrm{~B}-$ & 17 & $47^{c}(28)$ \\
\hline 3 & & $(\mathrm{HO})_{2} \mathrm{~B}-$ & 18 & $64^{c}$ \\
\hline 4 & & $(\mathrm{HO})_{2} \mathrm{~B}$ & 19 & 78 \\
\hline 5 & 11 & $(\mathrm{HO})_{2} \mathrm{~B}-$ & 20 & 70 \\
\hline 6 & & $(\mathrm{HO})_{2} \mathrm{~B}$ & 21 & $36(46)$ \\
\hline 7 & & $(\mathrm{HO})_{2} \mathrm{~B}-$ & 22 & 63 \\
\hline 8 & 13 & $(\mathrm{HO})_{2}$ & 23 & 61 \\
\hline 9 & & $(\mathrm{HO})_{2} \mathrm{~B}$ & 24 & 52 \\
\hline 10 & 14 & $(\mathrm{HO})_{2} \mathrm{~B}-$ & 25 & $40(21), 33^{e}$ \\
\hline 11 & 15 & $\begin{array}{l}(\mathrm{HO})_{2} \mathrm{~B} \\
\mathrm{R}=\mathrm{H}, 4-\mathrm{OMe}\end{array}$ & - & $\mathrm{ND}^{d}$ \\
\hline
\end{tabular}

${ }^{a}$ Yields are indicated as isolated compounds. ${ }^{b}$ SM starting material recovered. ${ }^{c}$ Reaction time of the second step $48 \mathrm{~h} .{ }^{d} \mathrm{ND}$ : Not detected. ${ }^{e}$ Reaction time of the second step $72 \mathrm{~h}$.

2,4,6-trisubstituted compounds. The reactivity of the three thioethers 18, 19, and 22 was explored in the presence of boronic acids, using an (het)arylboronic acid in the presence of $\mathrm{Pd}\left(\mathrm{PPh}_{3}\right)_{4}$ and copper(I) thiophene-2-carboxylate (CuTC) in THF at $100{ }^{\circ} \mathrm{C}$ under microwave irradiation. ${ }^{7}$ To our great 
Table 4 Exemplification of the Liebeskind-Srogl arylation in C-2

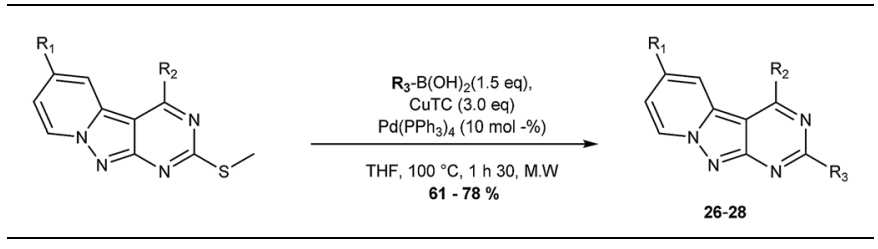

\begin{tabular}{|c|c|c|}
\hline \multicolumn{2}{|l|}{ Er } & Final compound \\
\hline
\end{tabular}
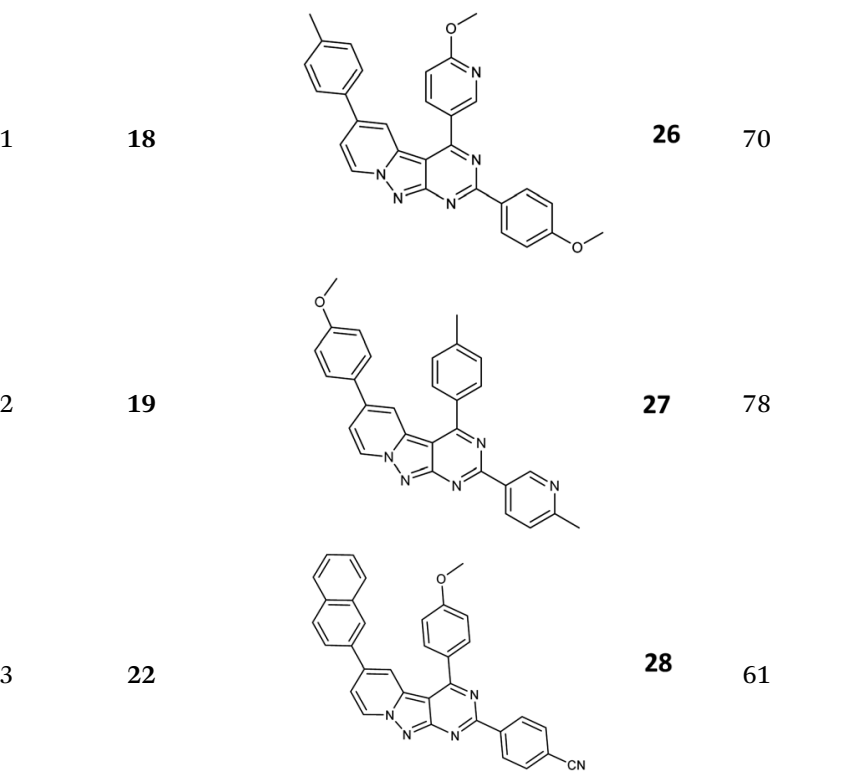

${ }^{a}$ Yields are indicated as isolated compounds.

satisfaction, the first test using 4-methoxyphenyl boronic acid as coupling partner gave $\mathbf{2 6}$ in a good $70 \%$ yield (Table 4 ). By changing both starting precursors (19 and 22) and the coupling partners, the tri-substituted tricycles 27 and 28 were readily isolated with $78 \%$ and $61 \%$ yields respectively (Table 4 ). The efficiency of this last reaction in C-2 seems insensitive to the nature of the 4,6-bis(het)arylated derivatives and boronic acids used.

\section{Conclusions}

In summary, we have described in this work several synthetic pathways for the preparation of a new pyrido $\left[1^{\prime}, 2^{\prime}: 1,5\right]$ pyrazolo $[3,4-d]$ pyrimidine scaffold, and have build several libraries of C2, C-4, C-6 (het)arylated pyrido $\left[1^{\prime}, 2^{\prime}: 1,5\right]$ pyrazolo[3,4- $\left.d\right]$ pyrimidines. First, the C-6 chlorine atom was substituted using a Suzuki-Miyaura cross coupling reaction. The resulting C-6arylated products were engaged in a direct $\mathrm{C}-\mathrm{O}$ activation from an amide function with PyBroP activation followed by a palladium catalyzed reaction to produce a series of C-4,6 bis(het)arylated compounds. We proved that electron criteria governed the reactivity and efficiency. Finally, the 2-SMe residual function reacted under Liebeskind-Srogl conditions to afford the desired tri-substituted derivatives. The efficiency of the global sequence involving three successive and unprecedented cross coupling reactions will certainly prove useful to others in the future to develop poly functionalized heterocyclic PPPy series.

\section{Experimental}

Reactions were monitored by thin-layer chromatography (TLC) analysis using silica gel (60 F254) plates. Compounds were visualized by UV irradiation. Flash column chromatography was performed on silica gel 60 (230-400.13 mesh, $0.0400 .063 \mathrm{~mm}$ ). Melting points ( $\mathrm{Mp}\left[{ }^{\circ} \mathrm{C}\right]$ ) were taken on samples in open capillary tubes and are uncorrected. The infrared spectra of compounds, recorded on a Thermo Scientific Nicolet is10, are given in $\mathrm{cm}^{-1}$. ${ }^{1} \mathrm{H}$ and ${ }^{13} \mathrm{C}$ NMR spectra were recorded on a Bruker Avance II $250\left({ }^{13} \mathrm{C}, 63 \mathrm{MHz}\right)$, Bruker Avance $400\left({ }^{13} \mathrm{C}\right.$, $101 \mathrm{MHz})$, or on a Bruker Avance III HD nanobay $400\left({ }^{13} \mathrm{C}, 101\right.$ $\mathrm{MHz}$ ). Chemical shifts are given in parts per million from tetramethylsilane (TMS) as internal standard. Coupling constants $(J)$ are reported in hertz $(\mathrm{Hz})$. High-resolution mass spectra (HRMS) were performed on a Maxis Bruker 4G.

\section{Characterization data}

1-Amino-4-chloro-pyridinium iodide 1:. ${ }^{9}$ In a $50 \mathrm{~mL}$ round bottom flask, 4-chloropyridine hydrochloride $(3.48 \mathrm{~g}$, $23.2 \mathrm{mmol}, 1.0$ eq.) was placed in water $(10 \mathrm{~mL})$. The solution was cooled at $0-5{ }^{\circ} \mathrm{C}$ under stirring, then sodium bicarbonate (4.48 g, $46.4 \mathrm{mmol}, 2.0 \mathrm{eq}$.) was added carefully over $10 \mathrm{~min}$. After $30 \mathrm{~min}$, the mixture was extracted with $\mathrm{CH}_{2} \mathrm{Cl}_{2}(3 \times 10$ $\mathrm{mL}$ ), dried over $\mathrm{Na}_{2} \mathrm{SO}_{4}$, and filtered. The filtrate was placed in a $50 \mathrm{~mL}$ round bottom flask, and a solution of $O$-(mesitylenesulfonyl)hydroxylamine (MSH) ${ }^{15}(5.0 \mathrm{~g}, 23.2 \mathrm{mmol}, 1.0 \mathrm{eq}$.$) in$ $\mathrm{CH}_{2} \mathrm{Cl}_{2}(10 \mathrm{~mL})$ was added (after drying over $\left.\mathrm{Na}_{2} \mathrm{SO}_{4}\right)$. The mixture was stirred overnight at room temperature under inert atmosphere and an aqueous $\mathrm{HI}$ solution was added ( $4 \mathrm{~mL}, 45 \%$ ) and stirred for $30 \mathrm{~min}$. The resulting solution was kept in a refrigerator to crystallize the desired compound $\mathbf{1}$ as orange crystals $(2.12 \mathrm{~g}, 35 \%)$. Mp $145-146{ }^{\circ} \mathrm{C} ; R_{\mathrm{f}}$ (petroleum ether: EtOAc, $4: 6$ ) = 0.1; IR (ATR diamond, $\mathrm{cm}^{-1}$ ) $\nu$ 833, 950, 1119, 1471, 1607, 3054, 3167; ${ }^{1} \mathrm{H}$ NMR $\left(400 \mathrm{MHz}\right.$, DMSO- $\left.d_{6}\right) \delta 8.18(\mathrm{~d}$, $J=7.0 \mathrm{~Hz}, 2 \mathrm{H}), 8.46\left(\mathrm{~s}, 2 \mathrm{H}, \mathrm{NH}_{2}\right), 8.76(\mathrm{~d}, J=7.0 \mathrm{~Hz}, 2 \mathrm{H}) ;{ }^{13} \mathrm{C}$ NMR (101 MHz, DMSO- $\left.d_{6}\right) \delta 128.2(2 \times \mathrm{CH}), 139.4(2 \times \mathrm{CH})$, 145.4 (Cq); HRMS (ESI) $[\mathrm{M}+\mathrm{H}]^{+}$calcd for $\mathrm{C}_{5} \mathrm{H}_{7} \mathrm{ClN}_{2}$ : 129.0214, found: 129.0214 .

Dimethyl 5-chloropyrazolo[1,5-a]pyridine-2,3-dicarboxylate 2. To a stirred solution of 1-amino-4-chloropyridin-1-ium iodide 1 (0.8 g, $3.11 \mathrm{mmol}, 1.0$ eq.) in DMF $(10 \mathrm{~mL})$ were added $\mathrm{K}_{2} \mathrm{CO}_{3}(0.6 \mathrm{~g}, 4.36 \mathrm{mmol}, 1.4$ eq. $)$ and dimethyl acetylenedicarboxylate (0.66 g, $4.6 \mathrm{mmol}, 1.5 \mathrm{eq}$.$) . The mixture was$ stirred at room temperature for $18 \mathrm{~h}$. The solvent was evaporated under reduced pressure, the residue was purified by flash chromatography (petroleum ether : EtOAc, $5: 5$ ) to give 2 as yellow crystals (523 mg, 86\%). Mp 120-121 ${ }^{\circ} \mathrm{C} ; R_{\mathrm{f}}$ (petroleum ether : EtOAc, $4: 6$ ) = 0.87; IR (ATR diamond, $\mathrm{cm}^{-1}$ ) $\nu 724,811$, 862, 1093, 1217, 1357, 1510, 1689, 3085; ${ }^{1} \mathrm{H}$ NMR $(400 \mathrm{MHz}$, $\left.\mathrm{CDCl}_{3}\right) \delta 3.94\left(\mathrm{~s}, 3 \mathrm{H}, \mathrm{CH}_{3}\right), 4.03\left(\mathrm{~s}, 3 \mathrm{H}, \mathrm{CH}_{3}\right), 7.02(\mathrm{dd}, J=7.3$, $2.3 \mathrm{~Hz}, 1 \mathrm{H}), 8.16(\mathrm{~d}, J=2.3 \mathrm{~Hz}, 1 \mathrm{H}), 8.43(\mathrm{~d}, J=7.3 \mathrm{~Hz}, 1 \mathrm{H}) ;{ }^{13} \mathrm{C}$ 
NMR (101 MHz, DMSO- $\left.d_{6}\right) \delta 51.8\left(\mathrm{CH}_{3}\right), 53.0\left(\mathrm{CH}_{3}\right), 102.7(\mathrm{Cq})$, $116.7(\mathrm{CH}), 118.7(\mathrm{CH}), 129.7(\mathrm{CH}), 135.2(\mathrm{Cq}), 141.5(\mathrm{Cq}), 148.1$ (Cq), 162.0 (CO), 162.7 (CO); HRMS (ESI) [M + H] calcd for $\mathrm{C}_{11} \mathrm{H}_{10} \mathrm{ClN}_{2} \mathrm{O}_{4}$ : 269.0324, found: 269.0321 .

5-Chloro-3-(methoxycarbonyl)pyrazolo[1,5-a]pyridine-2carboxylic acid 3. To a mixture of $2(0.5 \mathrm{~g}, 1.86 \mathrm{mmol}, 1.0$ eq.) in $\mathrm{MeOH}(60 \mathrm{~mL})$ was added $\mathrm{NaOH}(2 \mathrm{~N})(2.1 \mathrm{mmol})$ in one portion. The solution was stirred at room temperature for $6 \mathrm{~h}$, and then the solvent was removed. The residue was dissolved in $\mathrm{H}_{2} \mathrm{O}$ $(100 \mathrm{~mL})$ and the mixture was acidified to $\mathrm{pH} 1 \mathrm{using}$ a $10 \%$ aqueous $\mathrm{HCl}$ solution. The resulting solid was collected by filtration, washed with $\mathrm{Et}_{2} \mathrm{O}(20 \mathrm{~mL})$, and dried under reduced pressure to give $3(383 \mathrm{mg}, 81 \%)$ as a white solid. Mp 238$239{ }^{\circ} \mathrm{C} ; R_{\mathrm{f}}$ (petroleum ether : EtOAc, $2: 8$ ) $=0.1$; IR (ATR diamond, $\left.\mathrm{cm}^{-1}\right) \nu 664,764,856,1105,1209,1379,1480,1621$, 1750, 2651, 3083; ${ }^{1} \mathrm{H}$ NMR (400 MHz, $\left.\mathrm{CDCl}_{3}\right) \delta 4.15$ (s, 3H, $\mathrm{CH}_{3}$ ), $7.17(\mathrm{dd}, J=7.3,2.3 \mathrm{~Hz}, 1 \mathrm{H}), 8.14(\mathrm{~d}, J=2.3 \mathrm{~Hz}, 1 \mathrm{H}), 8.64(\mathrm{~d}, J=$ $7.3 \mathrm{~Hz}, 1 \mathrm{H}), 14.34$ (s, $1 \mathrm{H}, \mathrm{OH}) ;{ }^{13} \mathrm{C}$ NMR (101 MHz, DMSO- $\left.d_{6}\right)$ $\delta 52.1\left(\mathrm{CH}_{3}\right), 100.8(\mathrm{Cq}), 126.9(\mathrm{CH}), 127.8(\mathrm{CH}), 131.8(\mathrm{CH})$, 135.1 (Cq), 140.8 (Cq), 150.4 (Cq), 132.3 (CO), 134.2 (CO); HRMS (ESI) $[\mathrm{M}+\mathrm{H}]^{+}$calcd for $\mathrm{C}_{10} \mathrm{H}_{8} \mathrm{ClN}_{2} \mathrm{O}_{4}: 255.0167[\mathrm{M}+\mathrm{H}]^{+}$, found: 255.0164.

Methyl-2-((tert-butoxycarbonyl)amino)-5-chloropyrazolo[1,5a]pyridine-3-carboxylate 4. Starting from acid $3(1.0 \mathrm{~g}$, $3.92 \mathrm{mmol}, 1.0$ eq.), compound 4 was prepared following the procedure described in ref. 7 after purification by flash chromatography on silica gel (petroleum ether : EtOAc, $5: 5$ ) as a white solid $(0.857 \mathrm{~g}, 67 \%)$. Mp $188-190{ }^{\circ} \mathrm{C} ; R_{\mathrm{f}}$ (petroleum ether : EtOAc, $4: 6$ ) =0.8; IR (ATR diamond, $\mathrm{cm}^{-1}$ ) $\nu$ 779, 850, 1123, 1221, 1268, 1369, 1434, 1534, 1685, 2980, 3338; ${ }^{1} \mathrm{H}$ NMR $\left(400 \mathrm{MHz}, \mathrm{CDCl}_{3}\right) \delta 1.55\left(\mathrm{~s}, 9 \mathrm{H}, 3 \times \mathrm{CH}_{3}\right), 3.96\left(\mathrm{~s}, 3 \mathrm{H}, \mathrm{CH}_{3}\right), 6.86$ (dd, $J=7.3,2.3 \mathrm{~Hz}, 1 \mathrm{H}), 7.85(\mathrm{~d}, J=2.3 \mathrm{~Hz}, 1 \mathrm{H}), 8.44(\mathrm{~d}, J=$ $7.3 \mathrm{~Hz}, 1 \mathrm{H}), 8.97(\mathrm{~s}, 1 \mathrm{H}, \mathrm{NH}) ;{ }^{13} \mathrm{C} \mathrm{NMR}\left(101 \mathrm{MHz}, \mathrm{CDCl}_{3}\right) \delta 28.6$ $\left(3 \times \mathrm{CH}_{3}\right), 51.9\left(\mathrm{CH}_{3}\right), 82.1(\mathrm{Cq}), 90.0(\mathrm{Cq}), 114.9(\mathrm{CH}), 117.1$ (CH), 130.5 (CH), 135.9 (Cq), 140.8 (Cq), 151.1 (Cq), 154.1 (CO), 165.1 (CO); HRMS (ESI) $[\mathrm{M}+\mathrm{H}]^{+}$calcd for $\mathrm{C}_{14} \mathrm{H}_{17} \mathrm{ClN}_{3} \mathrm{O}_{4}$ : 326.0902 [M + H $]^{+}$, found: 326.0901 .

Methyl 2-amino-5-chloropyrazolo[1,5-a]pyridine-3-carboxylate 5. Starting from Boc-aminoester 4 ( $0.5 \mathrm{~g}, 1.53 \mathrm{mmol}, 1.0$ eq.), compound $\mathbf{5}$ was prepared following the procedure described in ref. 7 as a white solid in quantitative yield. Mp $196-197{ }^{\circ} \mathrm{C} ; R_{\mathrm{f}}$ (petroleum ether : EtOAc, $4: 6$ ) = 0.67; IR (ATR diamond, $\mathrm{cm}^{-1}$ ) $\nu$ 776, 864, 1009, 1112, 1280, 1437, 1620, 1678, 3104, 3422; ${ }^{1} \mathrm{H}$ NMR $\left(400 \mathrm{MHz} \mathrm{CDCl}_{3}\right) \delta 3.92\left(\mathrm{~s}, 3 \mathrm{H}, \mathrm{CH}_{3}\right), 5.26\left(\mathrm{~s}, 2 \mathrm{H}, \mathrm{NH}_{2}\right), 6.75(\mathrm{dd}, J$ $=7.3,2.3 \mathrm{~Hz}, 1 \mathrm{H}), 7.78(\mathrm{~d}, J=2.3 \mathrm{~Hz}, 1 \mathrm{H}), 8.11(\mathrm{~d}, J=7.3 \mathrm{~Hz}, 1 \mathrm{H})$; ${ }^{13} \mathrm{C}$ NMR (101 MHz, $\left.\mathrm{CDCl}_{3}\right) \delta 51.0\left(\mathrm{CH}_{3}\right) .87 .8(\mathrm{Cq}), 113.4(\mathrm{CH})$, $116.2(\mathrm{CH}), 128.7(\mathrm{CH}), 134.3(\mathrm{Cq}), 141.8(\mathrm{Cq}), 160.3(\mathrm{Cq}), 164.7$ (CO); HRMS (ESI) $[\mathrm{M}+\mathrm{H}]^{+}$calcd for $\mathrm{C}_{9} \mathrm{H}_{9} \mathrm{ClN}_{3} \mathrm{O}_{2}: 226.0378[\mathrm{M}+$ $\mathrm{H}]^{+}$, found: 226.0375 .

Methyl 2-(3-benzoylthioureido)-5-chloropyrazolo[1,5-a]pyridine3-carboxylate 6. Starting from aminoester 5 (1.0 g, $4.43 \mathrm{mmol}$, 1.0 eq.), compound 6 was prepared following the procedure described in ref. 7 after purification by flash chromatography on silica gel (petroleum ether: EtOAc, 4:6) as a yellow solid (1.44 g, 84\%). Mp 201-202 ${ }^{\circ} \mathrm{C} ; R_{\mathrm{f}}$ (petroleum ether : EtOAc, $4: 6$ ) $=0.90$; IR (ATR diamond, $\mathrm{cm}^{-1}$ ) $\nu$ 698, 807, 1006, 1112, 1156, 1303, 1435, 1529, 1678, 3040; ${ }^{1} \mathrm{H}$ NMR (400 MHz, $\left.\mathrm{CDCl}_{3}\right) \delta 4.05$ $\left(\mathrm{s}, 3 \mathrm{H}, \mathrm{CH}_{3}\right), 6.95(\mathrm{dd}, J=7.3,2.3 \mathrm{~Hz}, 1 \mathrm{H}), 7.59-7.47(\mathrm{~m}, 2 \mathrm{H})$, $7.71-7.62(\mathrm{~m}, 1 \mathrm{H}), 7.98(\mathrm{~d}, J=7.3 \mathrm{~Hz}, 2 \mathrm{H}), 8.04(\mathrm{~d}, J=2.3 \mathrm{~Hz}$, $1 \mathrm{H}), 8.49$ (d, $J=7.3 \mathrm{~Hz}, 1 \mathrm{H}), 9.27(\mathrm{~s}, 1 \mathrm{H}, \mathrm{NH}), 13.85(\mathrm{~s}, 1 \mathrm{H}, \mathrm{NH})$; ${ }^{13} \mathrm{C} \mathrm{NMR}\left(101 \mathrm{MHz}, \mathrm{CDCl}_{3}\right) \delta 52.2\left(\mathrm{CH}_{3}\right), 93.45(\mathrm{Cq}), 115.7(\mathrm{CH})$, $117.8(\mathrm{CH}), 128.1(2 \times \mathrm{CH}), 129.5(2 \times \mathrm{CH}), 130.6(\mathrm{Cq}), 131.9$ $(\mathrm{CH}), 134.1(\mathrm{Cq}), 136.1(\mathrm{CH}), 141.2(\mathrm{Cq}), 152.3(\mathrm{Cq}), 163.9(\mathrm{CO})$, 165.9 (CO), 176.6 (CS); HRMS (ESI) $[\mathrm{M}+\mathrm{H}]^{+}$calcd for $\mathrm{C}_{17} \mathrm{H}_{14^{-}}$ $\mathrm{ClN}_{4} \mathrm{O}_{3} \mathrm{~S}: 289.0470[\mathrm{M}+\mathrm{H}]^{+}$, found: 389.0468 .

6-Chloro-2-thioxo-2,3-dihydropyrido $\left[1^{\prime}, 2^{\prime}: 1,5\right]$ pyrazolo[3,4-d] pyrimidin-4(1H)-one 7. Starting from thiourea $6(1 \mathrm{~g}, 2.57$ $\mathrm{mmol}$ ), compound 7 was obtained following the procedure described in ref. 7 as a white solid $(0.63 \mathrm{~g}, 97 \%) . \mathrm{Mp}>360^{\circ} \mathrm{C} ; R_{\mathrm{f}}$ (petroleum ether : EtOAc, $4: 6$ ) =0.10; IR (ATR diamond, $\mathrm{cm}^{-1}$ ) $\nu$ 764, 824, 1106, 1208, 1379, 1480, 1621, 1750, 2652, 3083; ${ }^{1} \mathrm{H}$ NMR (400 MHz, DMSO- $d_{6}$ ) $\delta 7.36(\mathrm{dd}, J=7.3,2.3 \mathrm{~Hz}, 1 \mathrm{H}), 7.92$ $(\mathrm{d}, J=2.3 \mathrm{~Hz}, 1 \mathrm{H}), 8.95$ (d, $J=7.3 \mathrm{~Hz}, 1 \mathrm{H}), 12.15(\mathrm{~s}, 1 \mathrm{H}, \mathrm{NH})$, $13.36(\mathrm{~s}, 1 \mathrm{H}, \mathrm{NH}) ;{ }^{13} \mathrm{C}$ NMR (101 MHz, DMSO- $\left.d_{6}\right) \delta 92.8(\mathrm{Cq})$, $115.9(\mathrm{CH}), 116.9(\mathrm{CH}), 132.1(\mathrm{CH}), 135.6(\mathrm{Cq}), 138.6(\mathrm{Cq}), 154.4$ (Cq), 157.0 (CO), 177.0 (CS); HRMS (ESI) $[\mathrm{M}+\mathrm{H}]^{+}$calcd for $\mathrm{C}_{9^{-}}$ $\mathrm{H}_{6} \mathrm{ClN}_{4} \mathrm{OS}: 252.9945[\mathrm{M}+\mathrm{H}]^{+}$, found: 252.9942 .

6-Chloro-2-(methylthio)pyrido $\left[1^{\prime}, 2^{\prime}: 1,5\right]$ pyrazolo $[3,4-d]$ pyrimidin-4(3H)-one 8. Starting from compound $7(0.5 \mathrm{~g}$, $1.97 \mathrm{mmol}, 1.0$ eq.) and following the procedure described in ref. 7, with a reaction time adjusted to $6 \mathrm{~h}$, compound 8 was obtained as a white solid $(0.422 \mathrm{~g}, 80 \%)$. Mp $248-249{ }^{\circ} \mathrm{C} ; R_{\mathrm{f}}$ (petroleum ether : EtOAc, $4: 6$ ) $=0.57$; IR (ATR diamond, $\mathrm{cm}^{-1}$ ) $\nu$ 648, 782, 861, 1121, 1252, 1432, 1522, 1635, 2820, 3033; ${ }^{1} \mathrm{H}$ NMR (400 MHz, DMSO- $\left.d_{6}\right) \delta 2.58\left(\mathrm{~s}, 3 \mathrm{H}, \mathrm{CH}_{3}\right), 7.38(\mathrm{dd}, J=7.3$, $2.3 \mathrm{~Hz}, 1 \mathrm{H}), 8.00(\mathrm{~d}, J=2.3 \mathrm{~Hz}, 1 \mathrm{H}), 8.96(\mathrm{~d}, J=7.3 \mathrm{~Hz}, 1 \mathrm{H})$, 12.37 (s, $1 \mathrm{H}, \mathrm{NH}) ;{ }^{13} \mathrm{C}$ NMR $\left(101 \mathrm{MHz}, \mathrm{DMSO}-d_{6}\right) \delta 13.4\left(\mathrm{CH}_{3}\right)$, $94.8(\mathrm{Cq}), 116.6(\mathrm{CH}), 117.0(\mathrm{CH}), 131.6(\mathrm{CH}), 134.0(\mathrm{Cq}), 138.0$ (Cq), 158.7 (Cq), 161.9 (Cq), 162.4 (CO); HRMS (ESI) $[\mathrm{M}+\mathrm{H}]^{+}$ calcd for $\mathrm{C}_{10} \mathrm{H}_{8} \mathrm{ClN}_{4} \mathrm{OS}: 267.0102[\mathrm{M}+\mathrm{H}]^{+}$, found: 267.0100 .

\section{General procedure A for Suzuki-Miyaura cross coupling reaction}

A solution of compound 8 (100 $\mathrm{mg}, 0.37 \mathrm{mmol}, 1.0$ eq.) in 1,4dioxane $(4 \mathrm{~mL})$ was degassed by argon bubbling. Potassium carbonate (103 mg, $0.74 \mathrm{mmol}, 2.0$ eq.), boronic acid (1.1 eq.), xantphos (43 mg, $0.074 \mathrm{mmol}, 0.2$ eq.) and $\mathrm{Pd}(\mathrm{OAc})_{2}(8.0 \mathrm{mg}$, $0.037 \mathrm{mmol}, 0.1$ eq.) were then added and the reaction mixture was heated to $150{ }^{\circ} \mathrm{C}$ for $1 \mathrm{~h}$ under microwave irradiation. The crude material was concentrated under reduced pressure, and the residue was diluted in $\mathrm{CH}_{2} \mathrm{Cl}_{2}(10 \mathrm{~mL})$. The organic layers were washed with water $(10 \mathrm{~mL})$, dried over $\mathrm{MgSO}_{4}$, filtered and concentrated under reduced pressure. The crude residue was then purified by flash chromatography on silica to provide the desired compounds 10-15.

6. General procedure $B$ for the palladium catalyzed direct cross-coupling reactions involving a PyBroP-mediated $\mathrm{C}-\mathrm{O}$ activation

A suitable compound 10-11, 13-15 and PyBroP (1.2 eq.) were dissolved in 1,4-dioxane $(4 \mathrm{~mL})$ and $\mathrm{Et}_{3} \mathrm{~N}$ (3.0 eq.) was then added. Under stirring, the mixture was degassed by argon bubbling for $10 \mathrm{~min}$ and then heated at $80{ }^{\circ} \mathrm{C}$ for $18 \mathrm{~h}$. After 
cooling, the required (het)arylboronic acid (2.0 eq.), $\mathrm{Na}_{2} \mathrm{CO}_{3}$ (5.0 eq.) in $\mathrm{H}_{2} \mathrm{O}(1 \mathrm{~mL})$ and $\mathrm{PdCl}_{2}(\mathrm{dppf}) \cdot \mathrm{CH}_{2} \mathrm{Cl}_{2}(10 \mathrm{~mol} \%)$ were added. After heating at $110{ }^{\circ} \mathrm{C}$ for 24 hours generally, volatiles were removed under reduced pressure and the crude material diluted in $\mathrm{CH}_{2} \mathrm{Cl}_{2}(20 \mathrm{~mL})$. The combined organic extracts were dried over anhydrous $\mathrm{Na}_{2} \mathrm{SO}_{4}$, filtered, concentrated under reduced pressure, and purified by chromatography on silica gel to give the desired bis-arylated compounds 16-25.

\section{General procedure $\mathrm{C}$ for Liebeskind-Srogl cross-coupling reaction}

A microwave vial with THF $(4 \mathrm{~mL})$ under inert atmosphere was charged with the corresponding 4,6-bis(het)aryl-2-(methylthio)6-arylpyrido $\left[1^{\prime}, 2^{\prime}: 1,5\right]$ pyrazolo $[3,4-d]$ pyrimidines 18,19 or 22 , the corresponding aryl boronic acid (1.5 eq.), CuTC (3.0 eq.), and $\operatorname{Pd}\left(\mathrm{PPh}_{3}\right)_{4}(10 \mathrm{~mol} \%)$. The mixture was heated under microwave irradiation at $100{ }^{\circ} \mathrm{C}$ for $1 \mathrm{~h} 30 \mathrm{~min}$. After cooling to r.t., the solvent was removed under reduced pressure, the residue was diluted in aq. satd $\mathrm{NaHCO}_{3}$ solution $(10 \mathrm{~mL})$. The aqueous layer was extracted with $\mathrm{CH}_{2} \mathrm{Cl}_{2}(3 \times 10 \mathrm{~mL})$. The combined organic layers were dried with $\mathrm{MgSO}_{4}$, filtered, and concentrated under reduced pressure. The crude reaction mixture was purified by column chromatography on silica gel to provide the desired products 26-28.

2-(Methylthio)-4,6-di-p-tolylpyrido[ $\left[1^{\prime}, 2^{\prime}: 1,5\right]$ pyrazolo[3,4-d] pyrimidine 9. Starting from product $8(50 \mathrm{mg}, 0.18 \mathrm{mmol}, 1.0$ eq.) following the procedure $\mathbf{B}$, using $p$-tolylboronic acid ( $49 \mathrm{mg}$, $0.36 \mathrm{mmol}, 2.0$ eq.), compound $\mathbf{9}$ was obtained after purification by flash chromatography on silica gel (petroleum ether : EtOAc, $6: 4$ ) as a white solid (33 mg, 45\%). Mp 222-223 ${ }^{\circ} \mathrm{C} ; R_{\mathrm{f}}$ (petroleum ether : EtOAc, $4: 6$ ) $=0.80$; IR (ATR diamond, $\mathrm{cm}^{-1}$ ) $\nu 796$, 1005, 1163, 1244, 1452, 1578, 1641, 2919; ${ }^{1} \mathrm{H}$ NMR (400 MHz, $\left.\mathrm{CDCl}_{3}\right) \delta 2.42\left(\mathrm{~s}, 3 \mathrm{H}, \mathrm{CH}_{3}\right), 2.50\left(\mathrm{~s}, 3 \mathrm{H}, \mathrm{CH}_{3}\right), 2.74\left(\mathrm{~s}, 3 \mathrm{H}, \mathrm{CH}_{3}\right)$, $7.31(\mathrm{~d}, J=7.8 \mathrm{~Hz}, 2 \mathrm{H}), 7.41(\mathrm{~d}, J=7.8 \mathrm{~Hz}, 2 \mathrm{H}), 7.48(\mathrm{~d}, J=$ $7.8 \mathrm{~Hz}, 2 \mathrm{H}), 7.56-7.49(\mathrm{~m}, 1 \mathrm{H}), 7.86$ (d, $J=7.8 \mathrm{~Hz}, 2 \mathrm{H}), 8.16$ (s, $1 \mathrm{H}), 8.83(\mathrm{~d}, J=7.3 \mathrm{~Hz}, 1 \mathrm{H}) ;{ }^{13} \mathrm{C} \mathrm{NMR}\left(101 \mathrm{MHz}, \mathrm{CDCl}_{3}\right) \delta 14.5$ $\left(\mathrm{S}-\mathrm{CH}_{3}\right), 21.2\left(\mathrm{CH}_{3}\right), 21.6\left(\mathrm{CH}_{3}\right), 101.9(\mathrm{Cq}), 115.9(\mathrm{CH}), 116.8$ $(\mathrm{CH}), 126.7(2 \times \mathrm{CH}), 128.8(2 \times \mathrm{CH}), 129.5(2 \times \mathrm{CH}), 129.5(\mathrm{CH})$, $129.6(\mathrm{Cq}), 130.1(2 \times \mathrm{CH}), 134.6(\mathrm{Cq}), 134.7(\mathrm{Cq}), 136.0(\mathrm{Cq})$, 139.3 (Cq), 139.9 (Cq), 141.3 (Cq), 163.5 (Cq), 172.2 (Cq); HRMS (ESI) $[\mathrm{M}+\mathrm{H}]^{+}$calcd for $\mathrm{C}_{24} \mathrm{H}_{21} \mathrm{~N}_{4} \mathrm{~S}: 397.1481[\mathrm{M}+\mathrm{H}]^{+}$, found: 397.1481 .

2-(Methylthio)-6-( $p$-tolyl)pyrido $\left[1^{\prime}, 2^{\prime}: 1,5\right]$ pyrazolo[3,4- $\left.d\right]$ pyrimidin-4(3H)-one 10. Following the general procedure A using p-tolylboronic acid (57 mg, $0.41 \mathrm{mmol}, 1.1 \mathrm{eq}$.), compound 10 was obtained after purification by flash chromatography on silica gel (petroleum ether : EtOAc, $5: 5$ ) as a white solid (76 mg, $63 \%$ ). Mp $302-303{ }^{\circ} \mathrm{C} ; R_{\mathrm{f}}$ (petroleum ether : EtOAc, $\left.4: 6\right)=0.62$; IR (ATR diamond, $\mathrm{cm}^{-1}$ ) $\nu$ 666, 783, 873, 1001, 1122, 1210, 1363, 1447, 1580, 1643, 2829; ${ }^{1} \mathrm{H}$ NMR (400 MHz, DMSO- $\left.d_{6}\right) \delta 2.39$ (s, $\left.3 \mathrm{H}, \mathrm{CH}_{3}\right), 2.59\left(\mathrm{~s}, 3 \mathrm{H}, \mathrm{CH}_{3}\right), 7.36(\mathrm{~d}, J=7.8 \mathrm{~Hz}, 2 \mathrm{H}), 7.63(\mathrm{dd}, J=$ 7.3, $2.3 \mathrm{~Hz}, 1 \mathrm{H}), 7.87-7.70(\mathrm{~m}, 2 \mathrm{H}), 8.14(\mathrm{~d}, J=2.3 \mathrm{~Hz}, 1 \mathrm{H}), 8.95$ $(\mathrm{d}, J=7.3 \mathrm{~Hz}, 1 \mathrm{H}), 12.34(\mathrm{~s}, 1 \mathrm{H}, \mathrm{NH}) ;{ }^{13} \mathrm{C} \mathrm{NMR}(101 \mathrm{MHz}$, DMSO- $\left.d_{6}\right) \delta 13.4\left(\mathrm{CH}_{3}\right) .21 .2\left(\mathrm{~S}-\mathrm{CH}_{3}\right), 94.7(\mathrm{Cq}), 113.5(\mathrm{CH})$, $114.9(\mathrm{CH}), 127.2(2 \times \mathrm{CH}), 130.3(2 \times \mathrm{CH}), 130.4(\mathrm{CH}), 134.3$ (Cq), $138.1(\mathrm{Cq}), 139.3(\mathrm{Cq}), 140.1(\mathrm{Cq}), 158.8$ (Cq), 161.7 (Cq),
161.85 (CO); HRMS (ESI) $[\mathrm{M}+\mathrm{H}]^{+}$calcd for $\mathrm{C}_{17} \mathrm{H}_{15} \mathrm{~N}_{4} \mathrm{OS}$ : 323.0961 $[\mathrm{M}+\mathrm{H}]^{+}$, found: 323.0961.

6-(4-Methoxyphenyl)-2-(methylthio)pyrido[ $\left.1^{\prime}, 2^{\prime}: 1,5\right]$ pyrazolo $[3,4-d]$ pyrimidin-4(3H)-one 11 . Following the general procedure A using $p$-methoxyphenylboronic acid (62 $\mathrm{mg}, 0.41 \mathrm{mmol}, 1.1$ eq.), compound $\mathbf{1 1}$ was obtained after purification by flash chromatography on silica gel (petroleum ether : EtOAc, $5: 5$ ) as a white solid (92 mg, 73\%). Mp 295-296 ${ }^{\circ} \mathrm{C} ; R_{\mathrm{f}}$ (petroleum ether : EtOAc, $4: 6$ ) =0.50; IR (ATR diamond, $\mathrm{cm}^{-1}$ ) $\nu$ 956, 1033, 1113, 1251, 1453, 1518, 1661, 2835; ${ }^{1} \mathrm{H}$ NMR (400 MHz, DMSO$\left.d_{6}\right) \delta 2.59\left(\mathrm{~s}, 3 \mathrm{H}, \mathrm{CH}_{3}\right) .3 .84\left(\mathrm{~s}, 3 \mathrm{H}, \mathrm{CH}_{3}\right), 7.18-6.99(\mathrm{~m}, 2 \mathrm{H}), 7.61$ $(\mathrm{dd}, J=7.3,2.3 \mathrm{~Hz}, 1 \mathrm{H}), 7.91-7.76(\mathrm{~m}, 2 \mathrm{H}), 8.10(\mathrm{~d}, J=2.3 \mathrm{~Hz}$, $1 \mathrm{H}), 8.92(\mathrm{~d}, J=7.3 \mathrm{~Hz}, 1 \mathrm{H}), 12.31$ (s, $1 \mathrm{H}, \mathrm{NH}) ;{ }^{13} \mathrm{C}$ NMR $(101$ MHz, DMSO- $\left.d_{6}\right) \delta 12.9\left(\mathrm{~S}-\mathrm{CH}_{3}\right), 55.3\left(\mathrm{O}-\mathrm{CH}_{3}\right), 94.1(\mathrm{Cq}), 112.4$ $(\mathrm{CH}), 114.3(\mathrm{CH}), 114.7(2 \times \mathrm{CH}), 128.2(2 \times \mathrm{CH}), 128.9(\mathrm{Cq})$, $129.9(\mathrm{CH}), 137.7(\mathrm{Cq}), 139.4(\mathrm{Cq}), 158.3(\mathrm{Cq}), 160.1(\mathrm{Cq}), 161.2$ (Cq), 161.3 (CO); HRMS (ESI) $[\mathrm{M}+\mathrm{H}]^{+}$calcd for $\mathrm{C}_{17} \mathrm{H}_{15} \mathrm{~N}_{4} \mathrm{O}_{2} \mathrm{~S}$ : 339.0910 $[\mathrm{M}+\mathrm{H}]^{+}$, found: 339.0909.

6-(3-Methoxyphenyl)-2-(methylthio)pyrido $\left[1^{\prime}, 2^{\prime}: 1,5\right]$ pyrazolo $[3,4-d]$ pyrimidin-4(3H)-one 12 . Following the general procedure A using $m$-methoxyphenylboronic acid (62 mg, $0.41 \mathrm{mmol}, 1.1$ eq.), compound $\mathbf{1 2}$ was obtained after purification by flash chromatography on silica gel (petroleum ether : EtOAc, $5: 5$ ) as a white solid (86 mg, 68\%). Mp 266-267 ${ }^{\circ} \mathrm{C} ; R_{\mathrm{f}}$ (petroleum ether : EtOAc, $4: 6)=0.57$; IR (ATR diamond, $\mathrm{cm}^{-1}$ ) $\nu 775,863$, 1054, 1170, 1455, 1578, 1667, 2833; ${ }^{1} \mathrm{H}$ NMR (400 MHz, DMSO$\left.d_{6}\right) \delta 2.59\left(\mathrm{~s}, 3 \mathrm{H}, \mathrm{CH}_{3}\right), 3.87\left(\mathrm{~s}, 3 \mathrm{H}, \mathrm{CH}_{3}\right), 7.06(\mathrm{~d}, J=7.8 \mathrm{~Hz}, 1 \mathrm{H})$, $7.37(\mathrm{~s}, 1 \mathrm{H}), 7.41(\mathrm{~d}, J=7.8 \mathrm{~Hz}, 1 \mathrm{H}), 7.47$ (t, $J=7.8 \mathrm{~Hz}, 1 \mathrm{H}), 7.64$ (dd, $J=7.3,2.3 \mathrm{~Hz}, 1 \mathrm{H}), 8.16(\mathrm{~d}, J=2.3 \mathrm{~Hz}, 1 \mathrm{H}), 8.97$ (d, $J=$ $7.3 \mathrm{~Hz}, 1 \mathrm{H}), 12.35$ (s, 1H, NH); ${ }^{13} \mathrm{C}$ NMR (101 MHz, DMSO- $\left.d_{6}\right)$ $\delta 13.4\left(\mathrm{~S}-\mathrm{CH}_{3}\right), 55.7\left(\mathrm{O}-\mathrm{CH}_{3}\right), 94.9(\mathrm{Cq}), 112.2(\mathrm{CH}), 114.2(\mathrm{CH})$, $115.3(\mathrm{CH}), 115.4(\mathrm{CH}), 119.7(\mathrm{CH}), 130.4(\mathrm{CH}), 130.8(\mathrm{CH}), 138.0$ (Cq), $138.8(\mathrm{Cq}), 140.1(\mathrm{Cq}), 158.8(\mathrm{Cq}), 160.3(\mathrm{Cq}), 161.8(\mathrm{Cq})$, 161.9 (CO); HRMS (ESI) $[\mathrm{M}+\mathrm{H}]^{+}$calcd for $\mathrm{C}_{17} \mathrm{H}_{15} \mathrm{~N}_{4} \mathrm{O}_{2} \mathrm{~S}$ : 339.0910 $[\mathrm{M}+\mathrm{H}]^{+}$, found: 339.0907 .

2-(Methylthio)-6-(naphthalen-2-yl)pyrido[ $\left.1^{\prime}, 2^{\prime}: 1,5\right]$ pyrazolo $[3,4-d]$ pyrimidin-4(3H)-one 13 . Following the general procedure A using 2-naphthylboronic acid (71 mg, $0.41 \mathrm{mmol}, 1.1$ eq.), compound 13 was obtained after purification by flash chromatography on silica gel (petroleum ether : EtOAc, $5: 5$ ) as a white solid (94 mg, 70\%). Mp 311-312 ${ }^{\circ} \mathrm{C} ; R_{\mathrm{f}}$ (petroleum ether : EtOAc, $4: 6$ ) = 0.65; IR (ATR diamond, $\mathrm{cm}^{-1}$ ) $\nu$ 746, 803, 960, 1181, $1475,1582,1644,1739,3044 ;{ }^{1} \mathrm{H}$ NMR (400 MHz, DMSO- $d_{6}$ ) $\delta 2.60\left(\mathrm{~s}, 3 \mathrm{H}, \mathrm{CH}_{3}\right), 7.71-7.51(\mathrm{~m}, 2 \mathrm{H}), 7.80(\mathrm{dd}, J=7.3,2.3 \mathrm{~Hz}$, $1 \mathrm{H}), 8.00(\mathrm{dd}, J=9.9,6.2 \mathrm{~Hz}, 2 \mathrm{H}), 8.10(\mathrm{dd}, J=9.3,4.5 \mathrm{~Hz}, 2 \mathrm{H})$, $8.33(\mathrm{~d}, J=2.3 \mathrm{~Hz}, 1 \mathrm{H}), 8.49$ (d, $J=2.0 \mathrm{~Hz}, 1 \mathrm{H}), 9.03(\mathrm{~d}, J=$ $7.3 \mathrm{~Hz}, 1 \mathrm{H}), 12.38$ (s, $1 \mathrm{H}, \mathrm{NH}) ;{ }^{13} \mathrm{C}$ NMR (101 MHz, DMSO- $\left.d_{6}\right)$ $\delta 13.4\left(\mathrm{~S}-\mathrm{CH}_{3}\right), 94.9(\mathrm{Cq}), 114.3(\mathrm{CH}), 115.2(\mathrm{CH}), 124.9(\mathrm{CH})$, $126.8(\mathrm{CH}), 127.2(\mathrm{CH}), 127.45(\mathrm{CH}), 128.0(\mathrm{CH}), 129.05(\mathrm{CH})$, $129.4(\mathrm{CH}), 130.5(\mathrm{CH}), 133.4(\mathrm{Cq}), 133.6(\mathrm{Cq}), 134.5(\mathrm{Cq}), 138.1$ (Cq), 139.9 (Cq), 158.8 (Cq), 161.8 (CO), 161.9 (CO); HRMS (ESI) $[\mathrm{M}+\mathrm{H}]^{+}$calcd for $\mathrm{C}_{20} \mathrm{H}_{15} \mathrm{~N}_{4} \mathrm{OS}$ : $359.0961[\mathrm{M}+\mathrm{H}]^{+}$, found: 359.0959 .

6-(4-Fluorophenyl)-2-(methylthio)pyrido[ $\left.1^{\prime}, 2^{\prime}: 1,5\right]$ pyrazolo $[3,4-d]$ pyrimidin-4(3H)-one 14 . Following the general procedure A using $p$-fluorophenylboronic acid ( $57 \mathrm{mg}, 0.41 \mathrm{mmol}, 1.1$ eq.), compound $\mathbf{1 4}$ was obtained after purification by flash 
chromatography on silica gel (petroleum ether : EtOAc, $5: 5$ ) as a white solid (80 mg, 66\%). Mp 319-320 ${ }^{\circ} \mathrm{C} ; R_{\mathrm{f}}$ (petroleum ether : EtOAc, $4: 6$ ) = 0.62; IR (ATR diamond, $\mathrm{cm}^{-1}$ ) $\nu 658,784$, $870,1107,1233,1445,1647,2819 ;{ }^{1} \mathrm{H}$ NMR (400 MHz, DMSO- $\left.d_{6}\right)$ $\delta 2.59\left(\mathrm{~s}, 3 \mathrm{H}, \mathrm{CH}_{3}\right), 7.37(\mathrm{t}, J=8.6 \mathrm{~Hz}, 2 \mathrm{H}), 7.61(\mathrm{dd}, J=7.3$, $2.3 \mathrm{~Hz}, 1 \mathrm{H}), 7.99-7.88(\mathrm{~m}, 2 \mathrm{H}), 8.13$ (d, $J=2.3 \mathrm{~Hz}, 1 \mathrm{H}), 8.96$ (d, $J$ $=7.3 \mathrm{~Hz}, 1 \mathrm{H}), 12.34(\mathrm{~s}, 1 \mathrm{H}, \mathrm{NH}) ;{ }^{13} \mathrm{C}$ NMR $\left(101 \mathrm{MHz}, \mathrm{DMSO}-d_{6}\right)$ $\delta 13.4\left(\mathrm{~S}-\mathrm{CH}_{3}\right), 94.8(\mathrm{Cq}), 114.0(\mathrm{CH}), 115.0(\mathrm{CH}), 116.6\left(\mathrm{~d},{ }^{2} J_{\mathrm{C}-\mathrm{F}}\right.$ $=21.6 \mathrm{~Hz}, 2 \times \mathrm{CH}), 129.7\left(\mathrm{~d},{ }^{3} J_{\mathrm{C}-\mathrm{F}}=8.5 \mathrm{~Hz}, 2 \times \mathrm{CH}\right), 130.4(\mathrm{CH})$, $133.8\left(\mathrm{~d},{ }^{4} J_{\mathrm{C}-\mathrm{F}}=3.1 \mathrm{~Hz}, \mathrm{Cq}\right), 138.0(\mathrm{Cq}), 139.1(\mathrm{Cq}), 158.8(\mathrm{Cq})$, 161.8 (Cq), 161.8 (CO), 163.2 (d, $\left.{ }^{1} J_{\mathrm{C}-\mathrm{F}}=247 \mathrm{~Hz}, \mathrm{CF}\right)$; HRMS (ESI) $[\mathrm{M}+\mathrm{H}]^{+}$calcd for $\mathrm{C}_{16} \mathrm{H}_{12} \mathrm{FN}_{4} \mathrm{OS}$ : $327.0710[\mathrm{M}+\mathrm{H}]^{+}$, found: 327.0710 .

2-(Methylthio)-6-(4-(trifluoromethyl)phenyl)pyrido[ $\left[1^{\prime}, 2^{\prime}: 1,5\right]$ pyrazolo[3,4- $\boldsymbol{d}]$ pyrimidin-4(3H)-one 15 . Following the general procedure A using $p$-trifluorophenylboronic acid $(78 \mathrm{mg}$, $0.41 \mathrm{mmol}, 1.1 \mathrm{eq}$.), compound 15 was obtained after purification by flash chromatography on silica gel (petroleum ether: EtOAc, 5 : 5) as a white solid (79 mg, 56\%). $\mathrm{Mp} 342-343{ }^{\circ} \mathrm{C} ; R_{\mathrm{f}}$ (petroleum ether : EtOAc, $4: 6$ ) = 0.66; IR (ATR diamond, $\mathrm{cm}^{-1}$ ) $\nu 811,1015,1109,1330,1574,1650,2833,3044 ;{ }^{1} \mathrm{H}$ NMR $(400$ MHz, DMSO- $\left.d_{6}\right) \delta 2.58\left(\mathrm{~s}, 3 \mathrm{H}, \mathrm{CH}_{3}\right), 7.67(\mathrm{dd}, J=7.3,2.3 \mathrm{~Hz}$, $1 \mathrm{H}), 7.87(\mathrm{~d}, J=8.0 \mathrm{~Hz}, 2 \mathrm{H}), 8.08(\mathrm{~d}, J=8.0 \mathrm{~Hz}, 2 \mathrm{H}), 8.21(\mathrm{~d}, J=$ $2.3 \mathrm{~Hz}, 1 \mathrm{H}), 9.00(\mathrm{~d}, J=7.3 \mathrm{~Hz}, 1 \mathrm{H}), 12.38(\mathrm{~s}, 1 \mathrm{H}, \mathrm{NH}) ;{ }^{13} \mathrm{C}$ NMR (101 MHz, DMSO- $\left.d_{6}\right) \delta 12.9\left(\mathrm{~S}-\mathrm{CH}_{3}\right), 94.7(\mathrm{Cq}), 114.4(\mathrm{CH}), 114.5$ $(\mathrm{CH}), 124.1\left(\mathrm{q},{ }^{1} J_{\mathrm{C}-\mathrm{F} 3}=272 \mathrm{~Hz}, \mathrm{CF}_{3}\right), 126.0\left(\mathrm{q},{ }^{3} J_{\mathrm{C}-\mathrm{F} 3}=3.3 \mathrm{~Hz}, 2 \times\right.$ $\mathrm{CH}), 127.8(2 \times \mathrm{CH}), 129.4\left(\mathrm{q},{ }^{2} J_{\mathrm{C}-\mathrm{F} 3}=32 \mathrm{~Hz}, \mathrm{Cq}\right), 130.2(\mathrm{CH})$, $137.3(\mathrm{Cq}), 137.8$ (Cq), 140.8 (Cq), 158.3 (Cq), 161.4 (Cq), 161.5 (CO); HRMS (ESI) $[\mathrm{M}+\mathrm{H}]^{+}$calcd for $\mathrm{C}_{17} \mathrm{H}_{12} \mathrm{~F}_{3} \mathrm{~N}_{4} \mathrm{OS}$ : $377.0678[\mathrm{M}$ $+\mathrm{H}]^{+}$, found: 377.0676 .

4-(4-Methoxyphenyl)-2-(methylthio)-6-( $p$-tolyl)pyrido[ $\left[1^{\prime}, 2^{\prime}: 1,5\right]$ pyrazolo[3,4- $d]$ pyrimidine 16 . Starting from product $10(50 \mathrm{mg}$, $0.15 \mathrm{mmol}, 1.0 \mathrm{eq}$.), following the general procedure $\mathbf{B}$, using $p$ methoxyphenylboronic acid (47 $\mathrm{mg}, 0.31 \mathrm{mmol}, 2.0$ eq.), compound 16 was obtained after purification by flash chromatography on silica gel (petroleum ether : EtOAc, $6: 4$ ) as a yellow solid ( $35 \mathrm{mg}, 55 \%$ ). Mp $266-267^{\circ} \mathrm{C} ; R_{\mathrm{f}}$ (petroleum ether : EtOAc, $4: 6$ ) = 0.85; IR (ATR diamond, $\mathrm{cm}^{-1}$ ) $\nu 775,846,1023,1118$, 1444, 1577, 1653, 2976; ${ }^{1} \mathrm{H}$ NMR (400 MHz, $\left.\mathrm{CDCl}_{3}\right) \delta 2.43(\mathrm{~s}, 3 \mathrm{H}$, $\left.\mathrm{CH}_{3}\right), 2.74\left(\mathrm{~s}, 3 \mathrm{H}, \mathrm{CH}_{3}\right), 3.93\left(\mathrm{~s}, 3 \mathrm{H}, \mathrm{CH}_{3}\right), 7.12(\mathrm{~d}, J=8.5 \mathrm{~Hz}$, $2 \mathrm{H}), 7.32(\mathrm{~d}, J=7.8 \mathrm{~Hz}, 2 \mathrm{H}), 7.50(\mathrm{~d}, J=7.8 \mathrm{~Hz}, 2 \mathrm{H}), 7.56(\mathrm{dd}, J=$ $7.3 \mathrm{~Hz}, 1 \mathrm{H}), 7.95(\mathrm{~d}, J=8.5 \mathrm{~Hz}, 2 \mathrm{H}), 8.20(\mathrm{~s}, 1 \mathrm{H}), 8.85(\mathrm{~d}, J=$ $7.3 \mathrm{~Hz}, 1 \mathrm{H}) ;{ }^{13} \mathrm{C}$ NMR (101 MHz, $\left.\mathrm{CDCl}_{3}\right) \delta 14.6\left(\mathrm{~S}-\mathrm{CH}_{3}\right), 21.3$ $\left(\mathrm{CH}_{3}\right), 55.7\left(\mathrm{CH}_{3}\right), 101.9(\mathrm{Cq}), 114.4(2 \times \mathrm{CH}), 115.9(\mathrm{CH}), 117.1$ $(\mathrm{CH}), 126.9(2 \times \mathrm{CH}), 129.6(\mathrm{Cq}), 129.8(\mathrm{CH}), 130.3(2 \times \mathrm{CH})$, $130.7(2 \times \mathrm{CH}), 134.7(\mathrm{Cq}), 136.3(\mathrm{Cq}), 139.6(\mathrm{Cq}), 140.3(\mathrm{Cq})$, $162.2(\mathrm{Cq}), 162.9(\mathrm{Cq}), 163.5(\mathrm{Cq}), 171.8$ (Cq); HRMS (ESI) [M + $\mathrm{H}]^{+}$calcd for $\mathrm{C}_{24} \mathrm{H}_{21} \mathrm{~N}_{4} \mathrm{OS}: 413.1431[\mathrm{M}+\mathrm{H}]^{+}$, found: 413.1430 .

4-(4-Fluorophenyl)-2-(methylthio)-6-( $p$-tolyl)pyrido[ $\left.1^{\prime}, 2^{\prime}: 1,5\right]$ pyrazole[3,4- $\boldsymbol{d}]$ pyrimidine 17 . Starting from product $10(50 \mathrm{mg}$, $0.15 \mathrm{mmol}, 1.0 \mathrm{eq}$.), following the general procedure $\mathbf{B}$, using $p$ fluorophenylboronic acid (43 $\mathrm{mg}, 0.31 \mathrm{mmol}, 2.0 \mathrm{eq}$.), (reaction time of the second step $48 \mathrm{~h}$ ), compound 17 was obtained after purification by flash chromatography on silica gel (petroleum ether : EtOAc, $6: 4$ ) as a yellow solid (29 mg, 47\%). Mp 225$226{ }^{\circ} \mathrm{C} ; R_{\mathrm{f}}$ (petroleum ether : EtOAc, $4: 6$ ) $=0.89$; IR (ATR diamond, $\left.\mathrm{cm}^{-1}\right) \nu 799,870,1121,1450,1559,1636,1732,2921$;
${ }^{1} \mathrm{H}$ NMR $\left(400 \mathrm{MHz}, \mathrm{CDCl}_{3}\right) \delta 2.45\left(\mathrm{~s}, 3 \mathrm{H}, \mathrm{CH}_{3}\right), 2.76\left(\mathrm{~s}, 3 \mathrm{H}, \mathrm{CH}_{3}\right)$, $7.39-7.28(\mathrm{~m}, 4 \mathrm{H}), 7.50(\mathrm{~d}, J=7.8 \mathrm{~Hz}, 2 \mathrm{H}), 7.58(\mathrm{dd}, J=7.3$, $2.3 \mathrm{~Hz}, 1 \mathrm{H}), 8.02-7.96(\mathrm{~m}, 2 \mathrm{H}), 8.09$ (d, $J=2.3 \mathrm{~Hz}, 1 \mathrm{H}), 8.87$ (d, $J$ $=7.3 \mathrm{~Hz}, 1 \mathrm{H}) ;{ }^{13} \mathrm{C} \mathrm{NMR}\left(101 \mathrm{MHz}, \mathrm{CDCl}_{3}\right) \delta 14.4\left(\mathrm{CH}_{3}\right), 21.2(\mathrm{~S}-$ $\left.\mathrm{CH}_{3}\right), 101.8(\mathrm{Cq}), 115.5(\mathrm{CH}), 116.0\left(\mathrm{~d},{ }^{2} J_{\mathrm{C}-\mathrm{F}}=21.8 \mathrm{~Hz}, 2 \times \mathrm{CH}\right)$, $117.0(\mathrm{CH}), 126.7(2 \times \mathrm{CH}), 129.7(\mathrm{CH}), 130.2(2 \times \mathrm{CH}), 130.9(\mathrm{~d}$, $\left.{ }^{3} J_{\mathrm{C}-\mathrm{F}}=8.7 \mathrm{~Hz}, 2 \times \mathrm{CH}\right), 133.7\left(\mathrm{~d},{ }^{4} J_{\mathrm{C}-\mathrm{F}}=3.2 \mathrm{~Hz}, \mathrm{Cq}\right), 134.4(\mathrm{Cq})$, 135.8 (Cq), 139.5 (Cq), 140.2 (Cq), 162.2 (Cq), 163.4 (Cq), 164.4 $\left(\mathrm{d},{ }^{1} J_{\mathrm{C}-\mathrm{F}}=248 \mathrm{~Hz}, \mathrm{CF}\right), 172.2(\mathrm{Cq})$; HRMS (ESI) $[\mathrm{M}+\mathrm{H}]^{+}$calcd for $\mathrm{C}_{23} \mathrm{H}_{18} \mathrm{FN}_{4} \mathrm{~S}: 401.1231[\mathrm{M}+\mathrm{H}]^{+}$, found: 401.1231.

4-(6-Methoxypyridin-3-yl)-2-(methylthio)-6-(p-tolyl)pyrido $\left[1^{\prime}, 2^{\prime}: 1,5\right]$ pyrazolo $[3,4-d]$ pyrimidine 18 . Starting from product 10 (50 mg, $0.15 \mathrm{mmol}, 1.0$ eq.), following the general procedure $\mathbf{B}$, using 6-methoxy-3-pyridinylboronic acid ( $47 \mathrm{mg}, 0.31 \mathrm{mmol}, 2.0$ eq.), with a $48 \mathrm{~h}$ reaction time for the second step, compound 18 was obtained after purification by flash chromatography on silica gel (petroleum ether : EtOAc, 4:6) as a yellow solid (30 mg, 64\%). Mp $230-231^{\circ} \mathrm{C} ; R_{\mathrm{f}}$ (petroleum ether : EtOAc, $4: 6$ ) $=0.82$; IR (ATR diamond, $\mathrm{cm}^{-1}$ ) $\nu$ 798, 870, 1010, 1165, 1285, 1449, 1494, 1636, 2925; ${ }^{1} \mathrm{H}$ NMR $\left(400 \mathrm{MHz}, \mathrm{CDCl}_{3}\right) \delta 2.43(\mathrm{~s}, 3 \mathrm{H}$, $\left.\mathrm{CH}_{3}\right), 2.73\left(\mathrm{~s}, 3 \mathrm{H}, \mathrm{CH}_{3}\right), 4.07\left(\mathrm{~s}, 3 \mathrm{H}, \mathrm{CH}_{3}\right), 6.98(\mathrm{~d}, J=8.8 \mathrm{~Hz}$, $1 \mathrm{H}), 7.32(\mathrm{~d}, J=7.8 \mathrm{~Hz}, 2 \mathrm{H}), 7.59-7.49(\mathrm{~m}, 3 \mathrm{H}), 8.12(\mathrm{~s}, 1 \mathrm{H}), 8.19$ $(\mathrm{d}, J=8.8 \mathrm{~Hz}, 1 \mathrm{H}), 8.81(\mathrm{~d}, J=2.3 \mathrm{~Hz}, 1 \mathrm{H}), 8.85(\mathrm{~d}, J=7.3 \mathrm{~Hz}$, $1 \mathrm{H}) ;{ }^{13} \mathrm{C}$ NMR $\left(101 \mathrm{MHz}, \mathrm{CDCl}_{3}\right) \delta 14.6\left(\mathrm{CH}_{3}\right), 21.3\left(\mathrm{~S}-\mathrm{CH}_{3}\right), 54.1$ $\left(\mathrm{O}-\mathrm{CH}_{3}\right), 101.9(\mathrm{Cq}), 111.4(\mathrm{CH}), 115.6(\mathrm{CH}), 117.2(\mathrm{CH}), 126.9$ $(\mathrm{Cq}), 126.9(2 \times \mathrm{CH}), 129.8(\mathrm{CH}), 130.2(2 \times \mathrm{CH}), 134.5(\mathrm{Cq}), 135.9$ (Cq), $139.2(\mathrm{CH}), 139.6(\mathrm{Cq}), 140.5(\mathrm{Cq}), 147.9(\mathrm{CH}), 160.7(\mathrm{Cq})$, 163.5 (Cq), 165.9 (Cq), 172.3 (Cq); HRMS (ESI) $[\mathrm{M}+\mathrm{H}]^{+}$calcd for $\mathrm{C}_{23} \mathrm{H}_{20} \mathrm{~N}_{5} \mathrm{OS}: 414.1383[\mathrm{M}+\mathrm{H}]^{+}$, found: 414.1381 .

6-(4-Methoxyphenyl)-2-(methylthio)-4-( $p$-tolyl)pyrido[ $\left[1^{\prime}, 2^{\prime}: 1,5\right]-$ pyrazolo $[3,4-\boldsymbol{d}]$ pyrimidine 19 . Starting from product $11(50 \mathrm{mg}$, $0.147 \mathrm{mmol}, 1.0 \mathrm{eq}$.), following the general procedure $\mathbf{B}$, using p-tolylboronic acid (40 mg, $0.295 \mathrm{mmol}, 2.0 \mathrm{eq}$.), compound 19 was obtained after purification by flash chromatography on silica gel (petroleum ether : EtOAc, 4:6) as a yellow solid (47 mg, 78\%). Mp 209-210 ${ }^{\circ} \mathrm{C} ; R_{\mathrm{f}}$ (petroleum ether : EtOAc, $4: 6$ ) $=0.39$; IR (ATR diamond, $\mathrm{cm}^{-1}$ ) $\nu 724,1020,1117,1247,1452$, 1604, 1638, 2951; ${ }^{1} \mathrm{H}$ NMR (400 MHz, $\left.\mathrm{CDCl}_{3}\right) \delta 2.50\left(\mathrm{~s}, 3 \mathrm{H}, \mathrm{CH}_{3}\right)$, $2.73\left(\mathrm{~s}, 3 \mathrm{H}, \mathrm{CH}_{3}\right), 3.88\left(\mathrm{~s}, 3 \mathrm{H}, \mathrm{CH}_{3}\right), 7.06-6.99(\mathrm{~m}, 2 \mathrm{H}), 7.41(\mathrm{~d}, J$ $=7.8 \mathrm{~Hz}, 2 \mathrm{H}), 7.57-7.48(\mathrm{~m}, 3 \mathrm{H}), 7.90-7.84(\mathrm{~m}, 2 \mathrm{H}), 8.13(\mathrm{~d}, J=$ $2.2 \mathrm{~Hz}, 1 \mathrm{H}), 8.82(\mathrm{~d}, J=7.3 \mathrm{~Hz}, 1 \mathrm{H}) ;{ }^{13} \mathrm{C} \mathrm{NMR}\left(101 \mathrm{MHz}, \mathrm{CDCl}_{3}\right)$ $\delta 14.4\left(\mathrm{CH}_{3}\right), 21.6\left(\mathrm{~S}-\mathrm{CH}_{3}\right), 55.4\left(\mathrm{O}-\mathrm{CH}_{3}\right), 101.8(\mathrm{Cq}), 114.85(2 \times$ $\mathrm{CH}), 115.3(\mathrm{CH}), 116.6(\mathrm{CH}), 128.1(2 \times \mathrm{CH}), 128.8(2 \times \mathrm{CH})$, $129.5(2 \times \mathrm{CH}), 129.5(\mathrm{CH}), 129.7(\mathrm{Cq}), 134.7(\mathrm{Cq}), 136.0(\mathrm{Cq})$, 139.6 (Cq), $141.3(\mathrm{Cq}), 160.5(\mathrm{Cq}), 163.4(\mathrm{Cq}), 163.5(\mathrm{Cq}), 172.1$ (Cq); HRMS (ESI) $[\mathrm{M}+\mathrm{H}]^{+}$calcd for $\mathrm{C}_{24} \mathrm{H}_{21} \mathrm{~N}_{4} \mathrm{OS}$ : $413.1431[\mathrm{M}+$ $\mathrm{H}]^{+}$, found: 413.1428 .

4-[6-(4-Methoxyphenyl)-2-(methylthio)pyrido $\left[1^{\prime}, 2^{\prime}: 1,5\right]$ pyrazole[3,4- $\boldsymbol{d}]$ pyrimidin-4-yl]benzonitrile 20. Starting from product 11 (50 mg, $0.147 \mathrm{mmol}, 1.0$ eq.), following the general procedure B, using 4-cyanophenylboronic acid (43 mg, 0.295 mmol, 2.0 eq.), compound 20 was obtained after purification by flash chromatography on silica gel (petroleum ether: EtOAc, $4: 6$ ) as a yellow solid (62 mg, 70\%). $\mathrm{Mp}>360{ }^{\circ} \mathrm{C} ; R_{\mathrm{f}}$ (petroleum ether : EtOAc, $4: 6$ ) =0.59; IR (ATR diamond, $\mathrm{cm}^{-1}$ ) $\nu$ 813, 1037, 1164, 1244, 1452, 1572, 1637, 2227, 3044; ${ }^{1} \mathrm{H}$ NMR (400 MHz, DMSO- $\left.d_{6}\right) \delta 2.74\left(\mathrm{~s}, 3 \mathrm{H}, \mathrm{CH}_{3}\right), 3.89\left(\mathrm{~s}, 3 \mathrm{H}, \mathrm{CH}_{3}\right)$, 
7.10-7.00 (m, 2H), 7.52-7.45 (m, 2H), $7.58(\mathrm{dd}, J=7.3,2.3 \mathrm{~Hz}$, 1H), 7.97-7.89 (m, 3H), 8.13-8.05 (m, 2H), $8.86(\mathrm{~d}, J=7.3 \mathrm{~Hz}$, $1 \mathrm{H}) ;{ }^{13} \mathrm{C}$ NMR (101 MHz, DMSO- $\left.d_{6}\right) \delta 14.6\left(\mathrm{~S}-\mathrm{CH}_{3}\right), 55.6(\mathrm{O}-$ $\left.\mathrm{CH}_{3}\right), 101.8(\mathrm{Cq}), 114.65(\mathrm{Cq}), 115.0(\mathrm{CH}), 115.2(2 \times \mathrm{CH}), 117.3$ $(\mathrm{CH}), 118.4(\mathrm{Cq}), 128.2(2 \times \mathrm{CH}), 129.4(\mathrm{Cq}), 129.7(2 \times \mathrm{CH}), 130.0$ $(\mathrm{CH}), 132.8(2 \times \mathrm{CH}), 135.7(\mathrm{Cq}), 140.5(\mathrm{Cq}), 142.0(\mathrm{Cq}), 161.0$ (Cq), $161.2(\mathrm{Cq}), 163.5(\mathrm{Cq}), 172.6(\mathrm{Cq})$; HRMS (ESI) $[\mathrm{M}+\mathrm{H}]^{+}$ calcd for $\mathrm{C}_{24} \mathrm{H}_{18} \mathrm{~N}_{5} \mathrm{OS}: 424.1227[\mathrm{M}+\mathrm{H}]^{+}$, found: 424.1224 .

6-(4-Methoxyphenyl)-2-(methylthio)-4-(pyridin-3-yl)pyrido $\left[1^{\prime}, 2^{\prime}: \mathbf{1}, 5\right]$ pyrazolo $[3,4-d]$ pyrimidine 21 . Starting from product 11 (50 mg, $0.147 \mathrm{mmol}, 1.0$ eq.), following the general procedure $\mathbf{B}$, using 3-pyridinylboronic acid (36 mg, $0.295 \mathrm{mmol}, 2.0$ eq.), compound 21 was obtained after purification by flash chromatography on silica gel (petroleum ether : EtOAc, $4: 6$ ) as a yellow solid (21 mg, 36\%). Mp 222-223 ${ }^{\circ} \mathrm{C} ; R_{\mathrm{f}}$ (petroleum ether : EtOAc, $4: 6$ ) = 0.46; IR (ATR diamond, $\mathrm{cm}^{-1}$ ) $\nu$ 793, 1118, 1163, 1250, 1454, 1522, 1643, 2161, 2926; ${ }^{1} \mathrm{H}$ NMR (400 MHz, $\left.\mathrm{CDCl}_{3}\right) \delta 2.75$ $\left(\mathrm{s}, 3 \mathrm{H}, \mathrm{CH}_{3}\right), 3.87\left(\mathrm{~s}, 3 \mathrm{H}, \mathrm{CH}_{3}\right), 7.07-6.98(\mathrm{~m}, 2 \mathrm{H}), 7.64-7.50(\mathrm{~m}$, $4 \mathrm{H}), 7.99(\mathrm{~d}, J=2.3 \mathrm{~Hz}, 1 \mathrm{H}), 8.29(\mathrm{dd}, J=7.3,2.3 \mathrm{~Hz}, 1 \mathrm{H}), 8.86$ $(\mathrm{d}, J=7.3 \mathrm{~Hz}, 2 \mathrm{H}), 9.22(\mathrm{~s}, 1 \mathrm{H}) ;{ }^{13} \mathrm{C} \mathrm{NMR}\left(101 \mathrm{MHz}, \mathrm{CDCl}_{3}\right)$ $\delta 14.6\left(\mathrm{~S}-\mathrm{CH}_{3}\right), 55.6\left(\mathrm{O}-\mathrm{CH}_{3}\right), 102.1(\mathrm{Cq}), 115.0(\mathrm{CH}), 115.1(2 \times$ $\mathrm{CH}), 117.3(\mathrm{CH}), 124.0(\mathrm{CH}), 128.3(2 \times \mathrm{CH}), 129.5(\mathrm{Cq}), 129.9$ $(\mathrm{CH}), 135.9(\mathrm{Cq}), 133.7(\mathrm{Cq}), 136.6(\mathrm{CH}), 140.5(\mathrm{Cq}), 149.6(\mathrm{CH})$, $151.9(\mathrm{CH}), 160.4(\mathrm{Cq}), 160.9(\mathrm{Cq}), 163.4(\mathrm{Cq}), 172.5$ (Cq); HRMS (ESI) $[\mathrm{M}+\mathrm{H}]^{+}$calcd for $\mathrm{C}_{22} \mathrm{H}_{18} \mathrm{~N}_{5} \mathrm{OS}$ : $400.1227[\mathrm{M}+\mathrm{H}]^{+}$, found: 400.1226.

4-(4-Methoxyphenyl)-2-(methylthio)-6-(naphthalen-2-yl)pyrido $\left[1^{\prime}, \mathbf{2}^{\prime}: \mathbf{1}, 5\right]$ pyrazolo[3,4- $\left.d\right]$ pyrimidine 22 . Starting from product 13 (50 mg, $0.139 \mathrm{mmol}, 1.0$ eq.), following the general procedure $\mathbf{B}$, using 4-methoxyphenylboronic acid ( $42 \mathrm{mg}, 0.279 \mathrm{mmol}, 2.0 \mathrm{eq}$.), compound 22 was obtained after purification by flash chromatography on silica gel (petroleum ether : EtOAc, $5: 5$ ) as a yellow solid (39 mg, 63\%). Mp 271-272 ${ }^{\circ} \mathrm{C} ; R_{\mathrm{f}}$ (petroleum ether : EtOAc, $4: 6)=0.62$; IR (ATR diamond, $\mathrm{cm}^{-1}$ ) $\nu$ 763, 795, 841, 1031, 1168, $1245,1444,1555,1635,2933 ;{ }^{1} \mathrm{H}$ NMR $\left(400 \mathrm{MHz}, \mathrm{CDCl}_{3}\right) \delta 2.74(\mathrm{~s}$, $\left.3 \mathrm{H}, \mathrm{CH}_{3}\right) .3 .93\left(\mathrm{~s}, 3 \mathrm{H}, \mathrm{CH}_{3}\right), 7.14(\mathrm{~d}, J=8.4 \mathrm{~Hz}, 2 \mathrm{H}), 7.60-7.53(\mathrm{~m}$, 2H), 7.72-7.65 (m, 2H), 8.02-7.86 (m, 5H), 8.07 (s, 1H), 8.33 (s, $1 \mathrm{H}), 8.89$ (d, $J=7.0 \mathrm{~Hz}, 1 \mathrm{H}) ;{ }^{13} \mathrm{C}$ NMR $\left(101 \mathrm{MHz}, \mathrm{CDCl}_{3}\right) \delta 14.5$ $\left(\mathrm{CH}_{3}\right) .55 .5\left(\mathrm{CH}_{3}\right), 101.6(\mathrm{Cq}), 114.2(2 \times \mathrm{CH}), 116.4(\mathrm{CH}), 117.0$ $(\mathrm{CH}), 124.2(\mathrm{CH}), 126.4(\mathrm{CH}), 127.0(\mathrm{CH}), 127.1(\mathrm{CH}), 127.7(\mathrm{CH})$, $128.4(\mathrm{CH}), 129.3(\mathrm{CH}), 129.6(\mathrm{CH}), 129.9(\mathrm{Cq}), 130.6(2 \times \mathrm{CH})$, $133.2(\mathrm{Cq}), 133.4(\mathrm{Cq}), 134.7(\mathrm{Cq}), 136.0(\mathrm{Cq}), 139.7(\mathrm{Cq}), 162.0$ (Cq), $163.0(\mathrm{Cq}), 163.5(\mathrm{Cq}), 172.2(\mathrm{Cq})$; HRMS (ESI) $[\mathrm{M}+\mathrm{H}]^{+}$calcd for $\mathrm{C}_{27} \mathrm{H}_{21} \mathrm{~N}_{4} \mathrm{OS}$ : 449.1430 [M+ H] $]^{+}$, found: 449.1431.

2-[2-(Methylthio)-6-(naphthalen-2-yl)pyridoo[ $\left.1^{\prime}, 2^{\prime}: 1,5\right]$ pyrazolo$[3,4-d]$ pyrimidin-4-yl] benzonitrile 23 . Starting from product 13 (50 mg, $0.139 \mathrm{mmol}, 1.0$ eq.), following the general procedure $\mathbf{B}$, using 2-cyanophenylboronic acid (41 mg, $0.279 \mathrm{mmol}, 2.0$ eq.), compound 23 was obtained after purification by flash chromatography on silica gel (petroleum ether : EtOAc, $6: 4$ ) as a yellow solid (38 mg, 61\%). Mp 258-259 ${ }^{\circ} \mathrm{C} ; R_{\mathrm{f}}$ (petroleum ether : EtOAc, $4: 6$ ) = 0.80; IR (ATR diamond, $\mathrm{cm}^{-1}$ ) $\nu$ 726, 804, 908, 1124, $1165,1276,1445,1558,1637,2228,2925,3061 ;{ }^{1} \mathrm{H}$ NMR $(400$ $\left.\mathrm{MHz}, \mathrm{CDCl}_{3}\right) \delta 2.75\left(\mathrm{~s}, 3 \mathrm{H}, \mathrm{CH}_{3}\right), 7.61-7.53(\mathrm{~m}, 3 \mathrm{H}), 7.79-7.68$ $(\mathrm{m}, 3 \mathrm{H}), 8.03-7.82(\mathrm{~m}, 7 \mathrm{H}), 8.90(\mathrm{~d}, J=7.0 \mathrm{~Hz}, 1 \mathrm{H}) ;{ }^{13} \mathrm{C}$ NMR $\left(101 \mathrm{MHz}, \mathrm{CDCl}_{3}\right) \delta 14.5\left(\mathrm{CH}_{3}\right) \cdot 102.4(\mathrm{Cq}), 112.2(\mathrm{Cq}), 116.0$ $(\mathrm{CH}), 117.4(\mathrm{Cq}), 117.8(\mathrm{CH}), 124.1(\mathrm{CH}), 126.6(\mathrm{CH}), 127.0(\mathrm{CH})$,
$127.2(\mathrm{CH}), 127.7(\mathrm{CH}), 128.4(\mathrm{CH}), 129.3(\mathrm{CH}), 129.9(\mathrm{CH}), 130.0$ $(\mathrm{CH}), 130.6(\mathrm{CH}), 132.9(\mathrm{CH}), 133.3(\mathrm{Cq}), 133.7(\mathrm{Cq}), 134.1(\mathrm{CH})$, 134.5 (Cq), 135.7 (Cq), 140.4 (Cq), 140.6 (Cq), 159.4 (Cq), 163.1 (Cq), $172.6(\mathrm{Cq})$; HRMS (ESI) $[\mathrm{M}+\mathrm{H}]^{+}$calcd for $\mathrm{C}_{27} \mathrm{H}_{18} \mathrm{~N}_{5} \mathrm{~S}$ : 444.1277 $[\mathrm{M}+\mathrm{H}]^{+}$, found: 444.1278.

2-(Methylthio)-6-(naphthalen-2-yl)-4-(thiophen-2-yl)pyrido $\left[1^{\prime}, 2^{\prime}: 1,5\right]$ pyrazolo[3,4- $\left.d\right]$ pyrimidine 24 . Starting from product 13 (50 mg, $0.139 \mathrm{mmol}, 1.0 \mathrm{eq}$.), following the general procedure $\mathbf{B}$, using 2-thienylboronic acid (35 mg, $0.279 \mathrm{mmol}, 2.0$ eq.), compound $\mathbf{2 4}$ was obtained after purification by flash chromatography on silica gel (petroleum ether : EtOAc, $6: 4$ ) as a yellow solid (31 mg, 52\%). Mp $240-241{ }^{\circ} \mathrm{C} ; R_{\mathrm{f}}$ (petroleum ether : EtOAc, $4: 6$ ) = 0.90; IR (ATR diamond, $\mathrm{cm}^{-1}$ ) $\nu$ 793, 959, 1168, 1229, 1349, 1444, 1557, 1638, 2921, 3074; ${ }^{1} \mathrm{H}$ NMR (400 MHz, $\mathrm{CDCl}_{3}$ ) $\delta 2.71\left(\mathrm{~s}, 3 \mathrm{H}, \mathrm{CH}_{3}\right), 7.30(\mathrm{t}, J=4.2 \mathrm{~Hz}, 1 \mathrm{H}), 7.60-7.53(\mathrm{~m}, 2 \mathrm{H})$, $7.69(\mathrm{~m}, 2 \mathrm{H}), 7.74(\mathrm{~m}, 1 \mathrm{H}), 7.96-7.86(\mathrm{~m}, 2 \mathrm{H}), 8.04-7.96(\mathrm{~m}, 2 \mathrm{H})$, $8.11(\mathrm{~s}, 1 \mathrm{H}), 8.66(\mathrm{~s}, 1 \mathrm{H}), 8.88(\mathrm{~d}, J=7.1 \mathrm{~Hz}, 1 \mathrm{H}) ;{ }^{13} \mathrm{C} \mathrm{NMR}(101$ $\left.\mathrm{MHz}, \mathrm{CDCl}_{3}\right) \delta 14.4\left(\mathrm{CH}_{3}\right), 100.9(\mathrm{Cq}), 116.4(\mathrm{CH}), 117.2(\mathrm{CH})$, $124.2(\mathrm{CH}), 126.5(\mathrm{CH}), 127.0(\mathrm{CH}), 127.1(\mathrm{CH}), 127.7(\mathrm{CH}), 127.8$ $(\mathrm{CH}), 128.4(\mathrm{CH}), 129.3(\mathrm{CH}), 129.5(\mathrm{CH}), 129.7(\mathrm{CH}), 130.8(\mathrm{CH})$, $133.3(\mathrm{Cq}), 133.4(\mathrm{Cq}), 134.7$ (Cq), 135.7 (Cq), 140.0 (Cq), 141.3 (Cq), $156.4(\mathrm{Cq}), 163.6(\mathrm{Cq}), 171.9(\mathrm{Cq})$; HRMS (ESI) $[\mathrm{M}+\mathrm{H}]^{+}$ calcd for $\mathrm{C}_{24} \mathrm{H}_{17} \mathrm{~N}_{4} \mathrm{~S}_{2}$ : $425.0889[\mathrm{M}+\mathrm{H}]^{+}$, found: 425.0890 .

4-[6-(4-Fluorophenyl)-2-(methylthio)pyrido $\left[1^{\prime}, 2^{\prime}: 1,5\right]$ pyrazole [3,4- $\boldsymbol{d}]$ pyrimidin-4-yl]benzonitrile 25 . Starting from product 14 (50 mg, $0.153 \mathrm{mmol}, 1.0 \mathrm{eq}$.), following the general procedure $\mathbf{B}$, using 4-cyanophenylboronic acid (45 mg, $0.3 \mathrm{mmol}, 2.0$ eq.), compound 25 was obtained by purification by flash chromatography on silica gel (petroleum ether : EtOAc, $4: 6$ ) as a yellow solid (25 mg, 40\%). Mp 343-344 ${ }^{\circ} \mathrm{C} ; R_{\mathrm{f}}$ (petroleum ether : EtOAc, $4: 6$ ) = 0.43; IR (ATR diamond, $\mathrm{cm}^{-1}$ ) $\nu$ 817, 1048, 1166, 1448, 1574, 1732, 2229, 2918, 3079; ${ }^{1} \mathrm{H}$ NMR (400 MHz, DMSO- $d_{6}$ ) $\delta 2.65\left(\mathrm{~s}, 3 \mathrm{H}, \mathrm{CH}_{3}\right), 7.40(\mathrm{t}, J=8.6 \mathrm{~Hz}, 2 \mathrm{H}), 7.92-7.78(\mathrm{~m}, 2 \mathrm{H})$, $7.98-7.89(\mathrm{~m}, 1 \mathrm{H}), 8.02(\mathrm{~d}, J=2.3 \mathrm{~Hz}, 1 \mathrm{H}), 8.20(\mathrm{t}, J=6.8 \mathrm{~Hz}$, $4 \mathrm{H}), 9.25$ (d, $J=7.3 \mathrm{~Hz}, 1 \mathrm{H}) ;{ }^{13} \mathrm{C}$ NMR (101 MHz, DMSO- $\left.d_{6}\right)$ $\delta 14.2\left(\mathrm{CH}_{3}\right) \cdot 102.2(\mathrm{Cq}), 113.9(\mathrm{Cq}), 116.1(\mathrm{CH}), 116.8\left(\mathrm{~d},{ }^{2} J_{\mathrm{C}-\mathrm{F}}=\right.$ $21.8 \mathrm{~Hz}, 2 \times \mathrm{CH}), 118.5(\mathrm{CH}), 129.8\left(\mathrm{~d},{ }^{3} J_{\mathrm{C}-\mathrm{F}}=8.5 \mathrm{~Hz}, 2 \times \mathrm{CH}\right)$, $130.1(2 \times \mathrm{CH}), 131.05(\mathrm{CH}), 131.05(\mathrm{Cq}), 133.5(2 \times \mathrm{CH}), 133.65$ $\left(\mathrm{d}, J_{\mathrm{C}-\mathrm{F}}=3.1 \mathrm{~Hz}, \mathrm{Cq}\right), 135.3(\mathrm{Cq}), 139.1(\mathrm{Cq}), 141.6(\mathrm{Cq}), 161.5$ (Cq), $163.0(\mathrm{Cq}), 163.3\left(\mathrm{~d},{ }^{1} J_{\mathrm{C}-\mathrm{F}}=248.0 \mathrm{~Hz}, \mathrm{CF}\right), 171.1(\mathrm{Cq})$; HRMS (ESI) $[\mathrm{M}+\mathrm{H}]^{+}$calcd for $\mathrm{C}_{23} \mathrm{H}_{15} \mathrm{FN}_{5} \mathrm{~S}: 412.1027[\mathrm{M}+\mathrm{H}]^{+}$, found: 412.1024 .

2-(4-Methoxyphenyl)-4-(6-methoxypyridin-3-yl)-6-p-tolylpyrido[ $\left[1^{\prime}, 2^{\prime}: 1,5\right]$ pyrazolo[3,4- $\left.d\right]$ pyrimidine 26. Starting from 18 (50 mg, $0.12 \mathrm{mmol}, 1.0 \mathrm{eq}$.), using 4-methoxyphenylboronic acid (28 mg, $0.18 \mathrm{mmol}, 1.5$ eq.) as coupling reagent and following the general procedure $\mathbf{C}$, compound $\mathbf{2 6}$ was isolated by purification by flash chromatography on silica gel (petroleum ether: EtOAc, $4: 6$ ) as white solid (40 mg, 70\%). Mp 239-240 ${ }^{\circ} \mathrm{C} ; R_{\mathrm{f}}$ (petroleum ether : EtOAc, $4: 6$ ) $=0.50$; IR (ATR diamond, $\mathrm{cm}^{-1}$ ) $\nu 800,870,1019,1120,1287,1451,1493,1602,1712,2991 ;{ }^{1} \mathrm{H}$ NMR (400 MHz, $\left.\mathrm{CDCl}_{3}\right) \delta 2.43\left(\mathrm{~s}, 3 \mathrm{H}, \mathrm{CH}_{3}\right), 3.90\left(\mathrm{~s}, 3 \mathrm{H}, \mathrm{CH}_{3}\right)$, $4.09\left(\mathrm{~s}, 3 \mathrm{H}, \mathrm{CH}_{3}\right), 7.03(\mathrm{~d}, J=8.5 \mathrm{~Hz}, 3 \mathrm{H}), 7.32(\mathrm{~d}, J=7.8 \mathrm{~Hz}, 2 \mathrm{H})$, 7.60-7.50 (m, 3H), 8.22 (s, 1H), 8.33 (dd, $J=8.5,2.3 \mathrm{~Hz}, 1 \mathrm{H})$, $8.72(\mathrm{~d}, J=8.5 \mathrm{~Hz}, 2 \mathrm{H}), 8.99-8.87(\mathrm{~m}, 2 \mathrm{H}) ;{ }^{13} \mathrm{C}$ NMR $(101 \mathrm{MHz}$, $\left.\mathrm{CDCl}_{3}\right) \delta 21.2\left(\mathrm{CH}_{3}\right), 54.0\left(\mathrm{CH}_{3}\right), 55.3\left(\mathrm{CH}_{3}\right), 111.2(\mathrm{CH}), 113.7$ $(2 \times \mathrm{CH}), 115.7(\mathrm{CH}), 117.2(\mathrm{CH}), 126.8(2 \times \mathrm{CH}), 127.8(\mathrm{Cq})$, 
$129.6(\mathrm{CH}), 130.1(2 \times \mathrm{CH}), 130.6(2 \times \mathrm{CH}), 130.9(\mathrm{Cq}), 134.5(\mathrm{Cq})$, $135.5(\mathrm{Cq}), 139.1(\mathrm{CH}), 139.2(\mathrm{Cq}), 139.3(\mathrm{Cq}), 139.7(\mathrm{Cq}), 143.2$ (Cq), $147.9(\mathrm{CH}), 160.7(\mathrm{Cq}), 161.9(\mathrm{Cq}), 163.3(\mathrm{Cq}), 165.7(\mathrm{Cq})$; HRMS (ESI) $[\mathrm{M}+\mathrm{H}]^{+}$calcd for $\mathrm{C}_{29} \mathrm{H}_{24} \mathrm{~N}_{5} \mathrm{O}_{2}$ : $474.1925[\mathrm{M}+\mathrm{H}]^{+}$, found: 474.1921 .

6-(4-Methoxyphenyl)-2-(6-methylpyridin-3-yl)-4-( $p$-tolyl)pyrido $\left[1^{\prime}, 2^{\prime}: 1,5\right]$ pyrazolo[3,4-d]pyrimidine 27. Starting from 19 (50 mg, $0.12 \mathrm{mmol}, 1.0 \mathrm{eq}$.), using 6-methyl-3-pyridinyl boronic acid (25 mg, $0.18 \mathrm{mmol}, 1.5$ eq.) as coupling reagent and following the general procedure $\mathbf{C}$, compound 27 was isolated by purification by flash chromatography on silica gel (petroleum ether : EtOAc, $4: 6$ ) as yellow solid (43 mg, 78\%). Mp 207$208{ }^{\circ} \mathrm{C} ; R_{\mathrm{f}}$ (petroleum ether : EtOAc, $4: 6$ ) $=0.40$; IR (ATR diamond, $\left.\mathrm{cm}^{-1}\right) \nu 835,1033,1278,1455,1605,1731,2849,2918$; ${ }^{1} \mathrm{H}$ NMR $\left(400 \mathrm{MHz}, \mathrm{CDCl}_{3}\right) \delta 2.54\left(\mathrm{~s}, 3 \mathrm{H}, \mathrm{CH}_{3}\right), 2.71\left(\mathrm{~s}, 3 \mathrm{H}, \mathrm{CH}_{3}\right)$, $3.89\left(\mathrm{~s}, 3 \mathrm{H}, \mathrm{CH}_{3}\right), 7.04(\mathrm{~d}, J=8.4 \mathrm{~Hz}, 2 \mathrm{H}), 7.47(\mathrm{~d}, J=7.7 \mathrm{~Hz}, 2 \mathrm{H})$, 7.35 (d, $J=8.2 \mathrm{~Hz}, 1 \mathrm{H}), 7.58$ (d, $J=7.8 \mathrm{~Hz}, 3 \mathrm{H}), 7.99$ (d, $J=$ $7.7 \mathrm{~Hz}, 2 \mathrm{H}), 8.29$ (s, 1H), 9.09-8.89 (m, 2H), $9.86(\mathrm{~s}, 1 \mathrm{H}) ;{ }^{13} \mathrm{C}$ DEPT NMR (101 MHz, DMSO- $\left.d_{6}\right) \delta 21.6\left(\mathrm{CH}_{3}\right), 24.3\left(\mathrm{CH}_{3}\right), 55.5$ $\left(\mathrm{CH}_{3}\right), 114.9(2 \times \mathrm{CH}), 115.1(\mathrm{CH}), 115.8(\mathrm{CH}), 117.3(\mathrm{CH}), 128.2$ $(2 \times \mathrm{CH}), 129.0(2 \times \mathrm{CH}), 129.6(2 \times \mathrm{CH}), 129.7(\mathrm{CH}), 139.0(\mathrm{CH})$, $149.8(\mathrm{CH})$; HRMS (ESI) $[\mathrm{M}+\mathrm{H}]^{+}$calcd for $\mathrm{C}_{29} \mathrm{H}_{24} \mathrm{~N}_{5} \mathrm{O}: 458.1975$ $[\mathrm{M}+\mathrm{H}]^{+}$, found: 458.1977 .

4-[4-(4-Methoxyphenyl)-6-(naphthalen-2-yl)pyrido[ $\left[1^{\prime}, 2^{\prime}: 1,5\right]$ pyrazolo[3,4- $d]$ pyrimidin-2-yl]benzonitrile 28 . Starting from 22 (50 mg, $0.10 \mathrm{mmol}, 1.0$ eq.), using 4-cyanophenylboronic acid (24 mg, $0.16 \mathrm{mmol}, 1.5$ eq.) as coupling reagent and following the general procedure $\mathbf{C}$, compound $\mathbf{2 8}$ was isolated by purification by flash chromatography on silica gel (petroleum ether: EtOAc, $4: 6$ ) as white solid (34 mg, 61\%). Mp 329-330 ${ }^{\circ} \mathrm{C} ; R_{\mathrm{f}}$ (petroleum ether : EtOAc, $4: 6$ ) $=0.75$; IR (ATR diamond, $\mathrm{cm}^{-1}$ ) $\nu$ 802, 1026, 1119, 1254, 1510, 1605, 1685, 2224, 2918; ${ }^{1} \mathrm{H}$ NMR $\left(250 \mathrm{MHz}, \mathrm{DMSO}-d_{6}\right) \delta 3.90\left(\mathrm{~s}, 3 \mathrm{H}, \mathrm{CH}_{3}\right), 7.29(\mathrm{~d}, J=8.5 \mathrm{~Hz}, 2 \mathrm{H})$, $7.64-7.48(\mathrm{~m}, 2 \mathrm{H}), 7.81$ (d, $J=8.7 \mathrm{~Hz}, 1 \mathrm{H}), 8.22-7.88(\mathrm{~m}, 8 \mathrm{H})$, $8.35(\mathrm{~d}, J=7.2 \mathrm{~Hz}, 2 \mathrm{H}), 8.70(\mathrm{~d}, J=8.2 \mathrm{~Hz}, 2 \mathrm{H}), 9.28(\mathrm{~d}, J=$ $7.1 \mathrm{~Hz}, 1 \mathrm{H}$ ); HRMS (ESI) $[\mathrm{M}+\mathrm{H}]^{+}$calcd for $\mathrm{C}_{33} \mathrm{H}_{22} \mathrm{~N}_{5} \mathrm{O}: 504.1819$ $[\mathrm{M}+\mathrm{H}]^{+}$, found: 504.1820.

\section{Conflicts of interest}

No conflict of interest for authors.

\section{Acknowledgements}

The authors acknowledge the OMJ (Office Mediterranéen de la Jeunesse) for financial support to Rabia Belaroussi. The authors thank the Ligue Contre le Cancer and the Labex IRON (ANR-11LABX-0018-01) for supplies.

\section{Notes and references}

1 A. Akahane, H. Katayama, T. Mitsunaga, T. Kato, T. Kinoshita, Y. Kita, T. Kusunoki, T. Terai, K. Yoshida and Y. Shiokawa, J. Med. Chem., 1999, 42, 779-783.

2 B. A. Johns, K. S. Gudmundsson, E. M. Turner, S. H. Allen, V. A. Samano, J. A. Ray, G. A. Freeman, F. L. Boyd Jr,
C. J. Sexton, D. W. Selleseth, K. L. Creech and K. R. Moniri, Bioorg. Med. Chem., 2005, 13, 2397-2411.

3 (a) K. L. Stevens, D. K. Jung, M. J. Alberti, J. G. Badiang, G. E. Peckham, J. M. Veal, M. Cheung, P. A. Harris, S. D. Chamberlain and M. R. Peel, Org. Lett., 2005, 7, 47534756; (b) M. Cheung, P. A. Harris, J. G. Badiang, G. E. Peckham, S. D. Chamberlain, M. J. Alberti, D. K. Jung, S. S. Harris, N. H. Bramson, A. H. Epperly, S. A. Stimpson and M. R. Peel, Bioorg. Med. Chem. Lett., 2008, 18, 5428-5430.

4 T. Koike, T. Takai, Y. Hoashi, M. Nakayama, Y. Kosugi, M. Nakashima, S.-i. Yoshikubo, K. Hirai and O. Uchikawa, J. Med. Chem., 2011, 54, 4207-4218.

5 (a) W. Zhang, A. L. McIver, M. A. Stashko, D. DeRyckere, B. R. Branchford, D. Hunter, D. Kireev, M. J. Miley, J. Norris-Drouin, W. M. Stewart, M. Lee, S. Sather, Y. Zhou, J. A. Di Paola, M. Machius, W. P. Janzen, H. S. Earp, D. K. Graham, S. V. Frye and X. Wang, J. Med. Chem., 2013, 56, 9693-9700; (b) C. Ríos-Luci, R. Domínguez-Kelly, L. G. León, E. Díaz-Rodríguez, R. Freire, A. Pandiella, I. Cikotiene and J. M. Padrón, Bioorg. Med. Chem. Lett., 2011, 21, 6641-6645; (c) D. W. Porter, M. Bradley, Z. Brown, S. J. Charlton, B. Cox, P. Hunt, D. Janus, S. Lewis, P. Oakley, D. O'Connor, J. Reilly, N. Smith and N. J. Press, Bioorg. Med. Chem. Lett., 2014, 24, 3285-3290; (d) V. Sharma, N. Chitranshi and A. K. Agarwal, Int. J. Med. Chem., 2014, 31, 28-58.

6 (a) A. Tikad, S. Routier, M. Akssira and G. Guillaumet, Org. Biomol. Chem., 2009, 7, 5113-5118; (b) A. Tikad, S. Routier, M. Akssira, J.-M. Léger, C. Jarry and G. Guillaumet, Synthesis, 2009, 2379-2384; (c) S. Grosse, C. Pillard, S. Massip, J. M. Léger, C. Jarry, S. Bourg, P. Bernard and G. Guillaumet, Chem.-Eur. J., 2012, 18, 14943-14947; (d) Z. Tber, M.-A. Hiebel, A. El Hakmaoui, M. Akssira, G. Guillaumet and S. Berteina-Raboin, J. Org. Chem., 2015, 80, 6564-6573; (e) V. Prieur, M. D. Pujol and G. Guillaumet, Eur. J. Chem., 2015, 6547-6556; $(f)$ M. Naas, S. ElKazzouli, E. M. Essassi, M. Bousmina and G. Guillaumet, Org. Lett., 2015, 17, 4320-4323; (g) S. Grosse, C. Pillard, S. Massip, M. Marchivie, C. Jarry, P. Bernard and G. Guillaumet, J. Org. Chem., 2015, 80, 8539-8551; (h) M. Loubidi, C. Pillard, A. El Hakmaoui, P. Bernard, M. Akssira and G. Guillaumet, $R S C A d v ., 2016$, 6, 7229-7238; (i) M. El Hafi, M. Naas, M. Loubidi, J. Jouha, Y. Ramli, J. T. Mague, E. M. Essassi and G. Guillaumet, C. R. Chim., 2017, 20, 927-933.

7 R. Belaroussi, A. El Hakmaoui, M. Akssira, G. Guillaumet and S. Routier, Eur. J. Org. Chem., 2016, 3550-3558.

8 R. Belaroussi, A. El Bouakher, M. Marchivie, S. Massip, C. Jarry, A. El Hakmaoui, G. Guillaumet, S. Routier and M. Akssira, Synthesis, 2013, 45, 2557-2566.

9 J. D. Oslob, R. S. Mcdowell, R. Johnson, H. Yang, M. Evanchik, C. A. Zaharia, H. Cai and L. W. Hu, WO2014008197, 2014, Chem. Abstr., 2014, 160, 190128.

10 (a) F. U. Schmitz, V. W. F. Tai, R. Rai, C. D. Roberts, A. D. M. Abadi, S. Baskaran, I. Slobodov, J. Maung and M. L. Neitzel, WO2009023179, 2009, Chem. Abstr., 2009, 150, 260191; (b) Y. Feng, Y. T. Chen, H. Sessions, 
J. K. Mishra, S. Chowdhury, Y. Yin, P. Lograsso, J.-L. Luo, T. Bannister and T. Schroeter, WO2011050245, 2011, Chem. Abstr., 2011, 154, 540472; (c) M. Henrich, T. Weil, S. Mueller, V. Kauss, E. Erdmare and R. Zemribo, WO2009095253, 2009, Chem. Abstr., 2009, 151, 245654; (d) T.-S. Chung, X. Qiao and R. Liu, WO2006068626, 2006, Chem. Abstr., 2006, 145, 104617.

11 (a) F. A. Kang, Z. Sui and W. V. Murray, J. Am. Chem. Soc., 2008, 130, 11300-11302; (b) F. A. Kang, Z. Sui and W. V. Murray, Eur. J. Org. Chem., 2009, 461-479; (c) F. A. Kang, J. C. Lanter, C. Cai, Z. Sui and W. V. Murray, Chem. Commun., 2010, 46, 1347-1349; (d) E. Frérot, J. Coste, A. Pantaloni, M.-N. Dufour and P. Jouin, Tetrahedron, 1991, 47, 259-270; (e) J. Coste, E. Frerot, P. Jouin and B. Castro, Tetrahedron Lett., 1991, 32, 19671970; (f) J. Coste, E. Frerot and P. Jouin, J. Org. Chem., 1994, 59, 2437-2446.
12 R. Belaroussi, A. El Hakmaoui, N. Percina, A. Chartier, M. Marchivie, S. Massip, C. Jarry, M. Akssira, G. Guillaumet and S. Routier, Eur. J. Org. Chem., 2015, 4006-4017.

13 C. Copin, N. Henry, F. Buron and S. Routier, Eur. J. Org. Chem., 2012, 3079-3083.

14 (a) Q. Sun, F. Suzenet and G. Guillaumet, J. Org. Chem., 2010, 75, 3473-3476; (b) H. Prokopcová and C. O. Kappe, Angew. Chem., Int. Ed., 2009, 48, 2276-2286; (c) F.-A. Alphonse, F. Suzenet, A. Keromnes, B. Lebret and G. Guillaumet, Synlett, 2002, 447-450; (d) C. Kusturin, L. S. Liebeskind, H. Rahman, K. Sample, B. Schweitzer, J. Srogl and W. L. Neumann, Org. Lett., 2003, 5, 4349-4352.

15 J. Mendiola, J. A. Rincon, C. Mateos, J. F. Soriano, O. de Frutos, J. K. Niemeier and E. M. Davis, Org. Process Res. Dev., 2009, 13, 263-267. 